\title{
Formation, growth and instability of vortex pairs in an axisymmetric stagnation flow
}

\author{
Jinjun Wang ${ }^{1}$, Chong Pan ${ }^{1}$, Kwing-So Choi ${ }^{2} \dagger$, Lei Gao ${ }^{3}$ and Qi-Xiang Lian ${ }^{1}$ \\ ${ }^{1}$ Fluid Mechanics Key Laboratory of Education Ministry, Beijing University of Aeronautics and \\ Astronautics, 100191 Beijing, PR China \\ ${ }^{2}$ Faculty of Engineering, University of Nottingham, Nottingham NG7 2RD, UK \\ ${ }^{3}$ School of Mechanical and Aerospace Engineering, Nanyang Technological University, \\ Singapore 639798
}

(Received 4 January 2012; revised 26 February 2013; accepted 16 April 2013; first published online 23 May 2013)

The formation, growth and instability of a pair of counter-rotating vortices over a circular plate in the downstream of a thin fishing line were studied using particle image velocimetry and flow visualization. Initially, the vortex pair in an axisymmetric stagnation flow was small, but it grew steadily by accumulating the shear-layer vorticity of the wake before going through vortical instability. Two types of vortical development were observed in the present experiment. Type I was a common type of vortical development in an axisymmetric stagnation flow over a circular plate. Here, the circulation of the vortex pair increased linearly with time reflecting a constant flux of vorticity impinging on the plate wall. After the growth, the counter-rotating pair of vortices went through an antisymmetric deformation in the wall-normal direction while the vortex deformation was symmetric in the wall-parallel direction. This was remarkably similar to the short-wavelength elliptic instability of counter-rotating vortices in an open system. On the other hand, type II development of a vortex pair was a rare case, where the vortices grew for much longer duration than in type I cases. This initiated a breakdown of vortices before the residual vorticity moved away from the centre of the plate. It is considered that the disturbance due to vortical instability could be partially responsible for the unexpectedly high heat transfer rate in the stagnation region of bluff bodies that has been reported in the last half-century.

Key words: vortex dynamics, vortex instability, vortex interactions

\section{Introduction}

\subsection{Convective heat transfer in the stagnation region}

The rate of convective heat transfer from bluff bodies depends on turbulence characteristics in the free stream and the Reynolds number (Kestin 1966; Ames \& Moffat 1990; Barrett \& Hollingworth 2001; Bae, Lele \& Sung 2003). It has been

$†$ Email address for correspondence: kwing-so.choi@nottingham.ac.uk 
shown, for example, that an increase in free stream turbulence from 0.5 to $2.7 \%$ leads to an increase in heat transfer across a stagnation-point boundary layer by more than $40 \%$ (Kestin, Maeder \& Sogin 1961). Even a small vorticity present in the free stream may be sufficiently amplified to affect the mean flow structures over bluff bodies, enhancing the rate of heat transfer in the stagnation region (Sutera, Maeder \& Kestin 1963; Oo \& Ching 2002). Such an enhancement can be explained by the vorticity amplification mechanism (Sutera 1965; Sadeh \& Brauer 1980). Here, the vortical filaments resulting from the flow disturbances are stretched in the strain field around the bluff bodies, thereby intensifying the vorticity and associated velocity fluctuations (Oo \& Ching 2001).

When flow non-uniformity is introduced in the upstream of bluff bodies by placing screens or grids, a regular pattern of longitudinal vortices is observed in the stagnation region. This dramatically changes the heat transfer from bluff bodies (Morkovin 1979; Böttcher \& Wedemeyer 1989). The size of these vortices is determined by the wavelength of flow non-uniformity, although much greater vortices were also observed in the stagnation region of a solid parachute model by Lian \& Zhou (1989). Hodson \& Nagib (1977) and Lian \& Su (1994) placed a thin wire in the upstream of a rectangular cylinder, where a large, steady counter-rotating vortex pair was formed in the stagnation region.

It has long been known that the size of vortical disturbances is important in the formation and development of these vortices in the stagnation region (Kestin \& Wood 1970; Dhanak \& Stuart 1995). In a recent theoretical study of distortion of unsteady three-dimensional disturbances in a stagnation region of the Hiemenz boundary layer, Xiong \& Lele (2004) found that the amplification of incoming vorticity strongly depends on the ratio of the disturbance length scale to the boundary-layer thickness. They showed that the maximum amplification of disturbances takes place when the length-scale ratio is about two. Under this condition, the incoming vorticity was increased by a factor of five through vortex stretching. Bae, Lele \& Sung (2000) indicated in their numerical simulation that the corresponding wall heat transfer was maximized when the length-scale ratio takes a similar value. When the length-scale ratio is greater than this optimum value, however, the heat transfer is reduced as the amplified vortices in the stagnation region are detached from the wall. For a smaller length-scale ratio, on the other hand, the vortices are damped by the viscous effect of the boundary layer. In other words, the ratio between the disturbance length scale to the boundary-layer thickness indicates a balance between the vortex stretching and viscous dissipation (Xiong \& Lele 2004).

The formation and development of vortices over bluff body surfaces are not the result of stagnation flow instability. Rather, it is a forced response to the incoming disturbances (Morkovin 1979). Indeed, it was shown by Kestin \& Wood (1970) that the Hiemenz boundary layer is linearly stable to three-dimensional disturbances. This was supported by the experimental result of Hodson \& Nagib (1977), who studied the development of a vortex pair over a rectangular cylinder in the downstream of a thin wire wake. In a similar study, Lian \& Su (1994) showed that a pair of counter-rotating vortices grew in size by accumulating the vortex flux of the incoming wake. After the development, the pair of vortices was shed off to either edge of the square plate. Here, the time scale of vortex growth was much greater than the time scale of mean strain, suggesting that a different mechanism for vortex growth is in place. The antisymmetric behaviour of a pair of counter-rotating vortices after the growth also suggests that vortices may be undergoing a short-wavelength elliptic instability. 


\subsection{Short-wavelength elliptic instability}

The elliptic instability is a linear instability mechanism where three-dimensional flows can be generated within two-dimensional elliptic streamlines (Kerswell 2002). This instability mechanism has been found in a number of different flow configurations, including localized two-dimensional vortices in a strain field (Moore \& Saffman 1975) as well as unbound two-dimensional flows with elliptical vortex cores (Pierrehumbert 1986). Crow (1970) has analysed a counter-rotating pair of trailing vortices from aircraft, where he found a symmetric, long-wave elliptic instability bearing his name. This can often lead to a chain of vortex rings as the sinuous perturbations grow to connect the vortices. He also found short-wave elliptic instability during his analysis. This was later examined by Leweke \& Williamson (1998), who investigated the development of counter-rotating vortices created by impulsively closing two flat plates in a water tank. They found that the wavelength of the short-wavelength elliptic instability was twice the diameter of the vortex core, which is in good agreement with the result of direct numerical simulations (Laporte \& Corjon 2000). They also made an interesting observation on the phase relationship between the short-wave perturbations on the pair of counter-rotating vortices. While the vortex centres displace in the same direction normal to the symmetry plane of the pair of vortices, the displacement in the symmetry plane is out of phase. It was also shown that the vortex centrelines are inclined by approximately $45^{\circ}$, coinciding with the direction of maximum stretching associated with the strain field that vortices induce on each other (Leweke \& Williamson 1998). When an axial stretching is present, there is no steady solution to the three-dimensional instability of elliptical vortex. In this case, the vortex is time-dependent; the vorticity level must increase with time or viscous effects should be strong enough to counterbalance the increase in the vorticity level.

\subsection{Objectives of the present study}

Although the effect of free stream turbulence on the heat transfer enhancement in the stagnation region of bluff bodies has been studied in recent years, the behaviour of vortex development associated with the heat transfer has not been reported. One of our objectives is to elucidate the process of formation and development of a pair of vortices in an axisymmetric stagnation region when a thin wake shear layer impinges on a circular plate. Subsequent instability of the pair of counter-rotating vortices at the end of the vortex growth, as observed by Lian \& Su (1994), is also carefully investigated with a view to identify the underlining mechanism. By employing both qualitative and quantitative techniques, we are able to show that the growth of a pair of vortices is a result of the accumulation of wake vortices, but not simply a direct response of the boundary layer to the three-dimensional disturbance. We can also show that the antisymmetric cooperative deformation of counter-rotating vortices in the final stage of development is consistent with the behaviour of the short-wavelength elliptic instability (Leweke \& Williamson 1998; Laporte \& Corjon 2000).

\section{Experimental set-up}

Experiments were carried out in a recirculating water channel at Beijing University of Aeronautics and Astronautics. The channel test section measured $0.6 \mathrm{~m}$ wide, $0.6 \mathrm{~m}$ deep and $4.8 \mathrm{~m}$ long. It was made of Plexiglas panels to allow optical access of particle image velocimetry (PIV) measurements and flow visualization. The free stream velocity was kept at $U=0.066 \mathrm{~m} \mathrm{~s}^{-1}$ in the present test, where the turbulence level was $0.8 \%$. A $200 \mathrm{~mm}$ long Nylon fishing line of diameter $d_{0}=0.5 \mathrm{~mm}$ was 


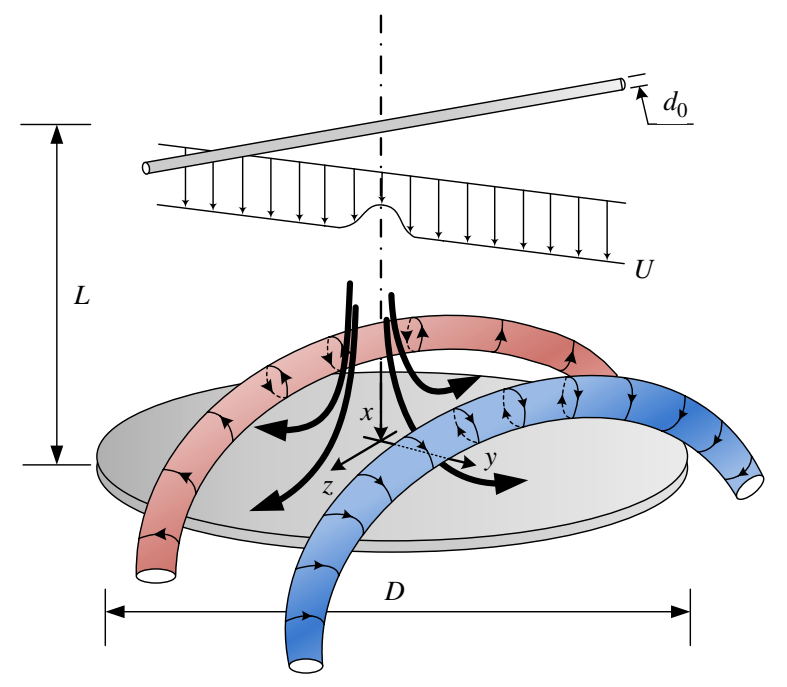

FiguRE 1. (Colour online) Sketch of the experimental set-up to show a pair of counterrotating vortices in an axisymmetric stagnation flow. A Nylon fishing line of diameter $d_{0}$ is stretched between two stainless steel prongs in a free stream of velocity $U$. The line was placed at a distance of $L$ upstream of a circular plate of diameter $D$ normal to the flow. Vortices are formed as a result of accumulated vorticity as the fishing-line wake impinges on the circular plate surface. The common flow direction of the vortices is away from the wall at the centre of a circular plate. The vortices are being stretched by the mean strain of diverging flow around a circular plate.

stretched between two stainless steel prongs. The line was placed at $L=50 \mathrm{~mm}(100$ $d_{0}$ ) upstream of a circular plate of diameter $D=60 \mathrm{~mm}$ normal to the flow. The Reynolds number of the flow about a fishing line was subcritical $(R e=34)$. The circular plate was made of $3 \mathrm{~mm}$ thick stainless steel, which was supported by a $10 \mathrm{~mm} \times 10 \mathrm{~mm}$ square rod, $200 \mathrm{~mm}$ long on the back (in the downstream). Before the test was conducted, the fishing line was carefully positioned so that its wake impinged on the surface of the circular plate exactly at the centre. The experimental set-up is schematically shown in figure 1 . We used the hydrogen bubble technique with a platinum wire $0.02 \mathrm{~mm}$ in diameter to visualize the stagnation flow. Here, the platinum wire was placed perpendicular to the fishing line. The corresponding Reynolds number based on the platinum wire and the free stream velocity was very small $(R e=2)$, therefore its effect on the vortical flow was negligible as compared with that created by a fishing line. The time lines of hydrogen bubbles were produced by applying a pulsing voltage to the wire at a frequency of $12 \mathrm{~Hz}$, allowing a detailed observation of vortical structures to be made with sufficient time and spatial resolution. The visualized flow patterns were captured using a digital video recorder at a frame rate of 25 frames per second (f.p.s.).

Velocity measurements in the stagnation flow over a circular plate was carried out using a time-resolved PIV system, consisting of a high-speed CCD camera (IMPERX ICL-B0620) of $640 \times 480$ pixels with Nikon $45 \mathrm{~mm}$ f2.8 lens. We used a $1.4 \mathrm{~W}$ continuous semiconductor laser (SM-SEMI-1400) of $532 \mathrm{~nm}$ wavelength. Images were sampled at $140 \mathrm{~Hz}$ with an exposure time of $2 \mathrm{~ms}$, where a pair of images was obtained at a frequency of $70 \mathrm{~Hz}$. Seeding particles (hollow glass beads 
of $1.05 \mathrm{~g} \mathrm{~cm}^{-3}$ in density with a median diameter of $5 \mu \mathrm{m}$ ) were added to the water channel before the measurement. The experimental condition for PIV measurements was identical to that of the flow visualization test described above, except that the diameter of a fishing line was $d_{0}=0.33 \mathrm{~mm}(R e=22)$, placed at $L=60 \mathrm{~mm}$ upstream of the circular plate $\left(180 d_{0}\right)$. PIV image processing was based on the progressive grid refinement technique (Scarano \& Riethmuller 2000), where the final interrogation was carried out in $16 \times 16$ pixel windows with $50 \%$ overlap. This gave a spatial resolution of $0.65 \mathrm{~mm}$ per velocity vector. Typical error in the resultant velocity was less than $1 \%$.

To identify vortices in the stagnation flow region, we used the spanwise vorticity as the identification criteria. Here, the threshold value was set to $3.5 \%$ of the wake shear rate, $S_{w}=U / d_{0}$, of a fishing line. The size and location of the vortices were then obtained by fitting circles to the contours of vorticity concentration region using a nonlinear, least-squares fitting algorithm (Coleman \& Li 1996). The circulation of vortices was obtained by integrating the vorticity within these circles. A detailed discussion on the present vortex identification technique in comparison with other techniques is given in Appendix. Error analysis of vortex parameters is also presented there.

\section{Characteristics of the base flow}

The radial and streamwise velocity contours in the upstream of a circular plate without a fishing line are shown in figure $2(a, b)$, respectively, at $x / D=$ $-0.2,-0.15,-0.1$ and -0.05 , based on the PIV measurements on 14 wall-normal planes. Despite the difficulty in measuring the vanishing flow velocities near the stagnation point, the axisymmetry of the base flow is well demonstrated in these figures.

Figure 3(a) shows the time-averaged streamlines over a circular plate without a fishing line. It also shows that a strong shear layer develops close to the plate wall, whose magnitude increases in the radial direction to reach the maximum value of $0.24\left(U / d_{0}\right)$ at the edge. Corresponding velocity and vorticity flow fields in the downstream of a fishing line are shown in figure $3(b)$, which were obtained by time-averaging 15000 PIV frames containing 43 continuous vortex development periods. Both figures demonstrate that the time-averaged flow field over a circular plate is symmetric without or with a fishing line. Figure 3(c) presents the mean velocity distribution along the frontal surface of the circular plate, showing the radial development of the boundary layer in a symmetry plane $(z=0)$ without a fishing line. The boundary-layer thickness was obtained from this figure as the location of maximum radial velocity, to give $\delta=0.030 \pm 0.005 D$. Superimposed in this figure is a distribution of non-dimensional vorticity, $\omega_{z}^{*}=\omega_{z} /\left(U / d_{0}\right)$, indicating that the concentration of vorticity is found only within the boundary layer as expected. Figure 3(c) also shows that the level of vorticity increases with an increase in the boundary-layer velocity towards the edge of the circular plate.

The wake velocity profile of a fishing line at $x / D=0$ without a circular plate is given in figure 4(a), confirming a symmetric velocity profile of a fishing-line wake at the location of a circular plate. The wake has a maximum velocity deficit of $0.09 U_{\infty}$ and a wake half-width of $0.06 \mathrm{D}$. The corresponding vorticity profile is shown in figure $4(b)$, giving the same wake width of $0.06 D$ based on the peak-to-peak distance. 

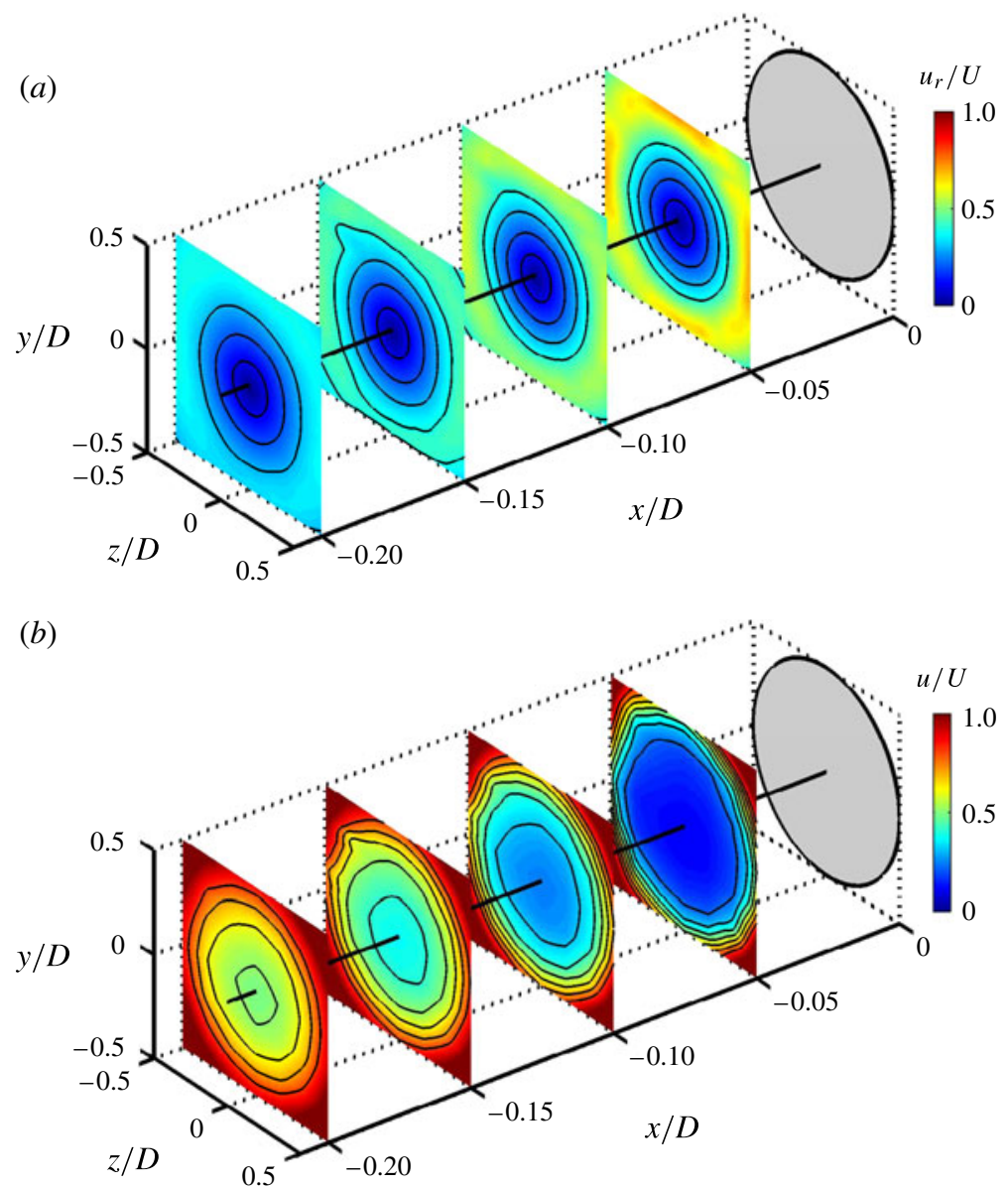

FIgURE 2. (Colour online) Time-averaged velocity distribution of the baseline flow around a circular plate: $(a)$ radial component $U_{r} / U$ and $(b)$ streamwise component $u / U$. The nondimensional velocity contour lines have a step of 0.1 .

\section{Development of a vortex pair}

The Reynolds number based on the diameter of the fishing line and the free stream velocity is subcritical, therefore no vortex shedding is expected in the laminar wake of the fishing line. This was confirmed by the flow visualization of the wake, as shown in figure $4(c)$. Figure $4(d)$ depicts the formation of a pair of counter-rotating vortices in the stagnation flow region, whose common flow direction in the centre of a circular plate is away from the wall. These vortices are formed as a result of accumulated vorticity as the wake shear layer impinges on the circular plate surface. A similar pair of counter-rotating vortices was observed by Hodson \& Nagib (1977) and Lian \& Su (1994) when a thin wire was placed in the upstream of a rectangular cylinder. It should be noted that the present study is fundamentally different from the investigation of Orlandi (1990) and Danaila (2004), who studied the behaviour of dipoles impinging on a wall. For example, the rotational direction of the vortex pair is opposite between the dipole studies and the present investigation. 

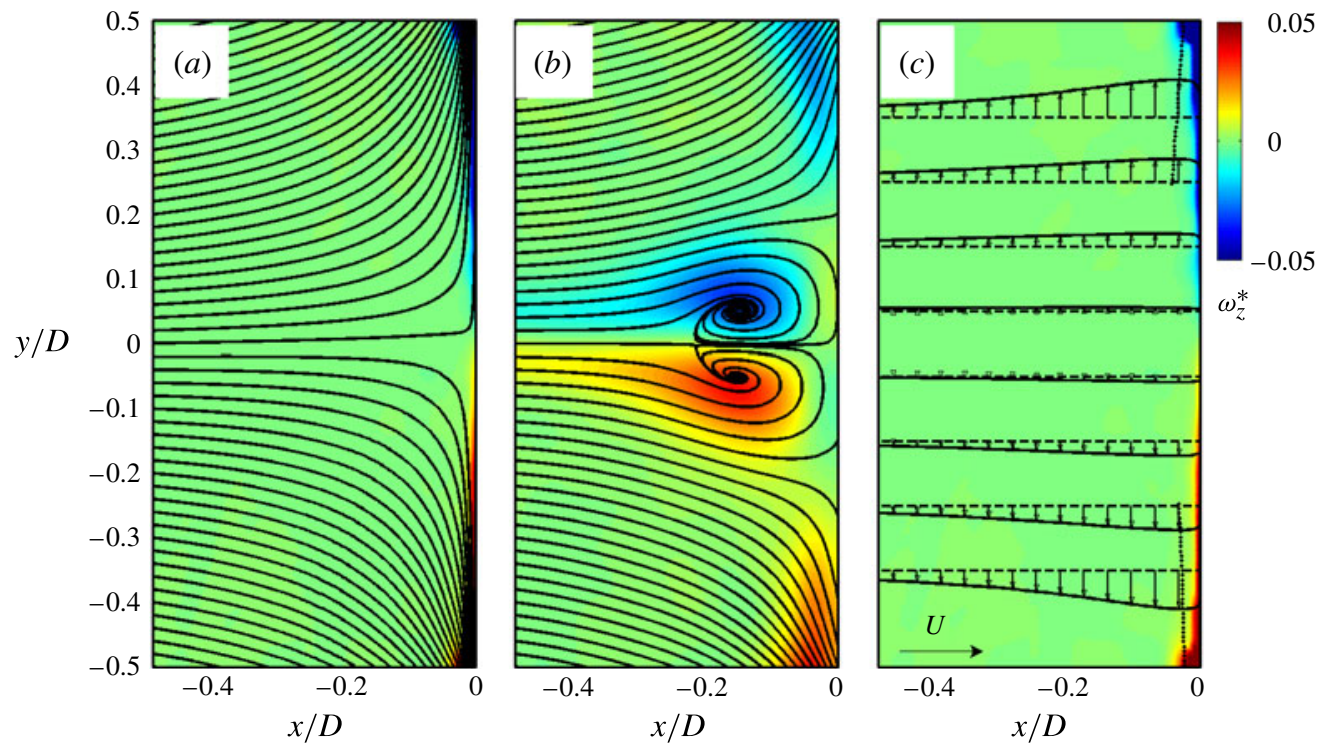

FIGURE 3. (Colour online) Time-averaged streamlines of stagnation flow over a circular plate without a fishing line $(a)$ and in the downstream of a fishing line $(b)$. Mean velocity distribution along the front surface of a circular plate $(c)$, showing the radial development of a boundary layer in a symmetry plane $(z=0)$ without a fishing line. The boundarylayer thickness is indicated by a dotted line. The distribution of the time-averaged spanwise vorticity is shown in a colour scale.

Two types of vortical development were observed in the present experiment. Type I is a common type of vortical development in an axisymmetric stagnation flow over a circular plate. Most of the vortical developments belong to type I, which can be further subdivided into type I-A and type I-B depending on the direction of wall-parallel movement of a vortex pair in the late stage. Type I-A is the vortex development where a pair of vortices moves in the positive $y$ direction after the vortex growth, while a vortex pair moves in the negative $y$ direction in type I-B development. Here, the flow field is symmetric with $y=0$ and the direction of the $y$-coordinate is arbitrary chosen, therefore type I-A and type I-B vortex development mechanisms are essentially the same. Indeed, there was no preferred direction to which pairs of vortices moved in the present experiment. The numbers of occurrence for type I-A and type I-B developments were also nearly the same. Type II development of a vortex pair, on the other hand, is a rare case, which was observed only in less than $5 \%$ of the total cases. In type II vortical development, vortices grow for a much longer duration than in type I cases. This initiated a breakdown of vortices before the residual vorticity moved away from the centre of the plate.

From PIV measurements, the averaged vortex shedding frequency behind a circular plate was found to be $0.91 \mathrm{~Hz}$, corresponding to the Strouhal number $S_{t}=0.82$. This corresponds to the non-dimensional time of $t^{*}=1.1$. Here, the non-dimensional time is defined by $t^{*}=t \Gamma / 2 \pi b^{2}$, where $\Gamma$ is the maximum circulation and $b$ is the maximum core spacing of the vortices during the development. The proportionality constant $\Gamma / 2 \pi b^{2}$ takes a value of about 1.0 in the present test. As will be shown later, the time scale of type I and type II development is much greater than that of vortex shedding from a circular plate. Therefore, it is highly unlikely that the vortex movement at the 

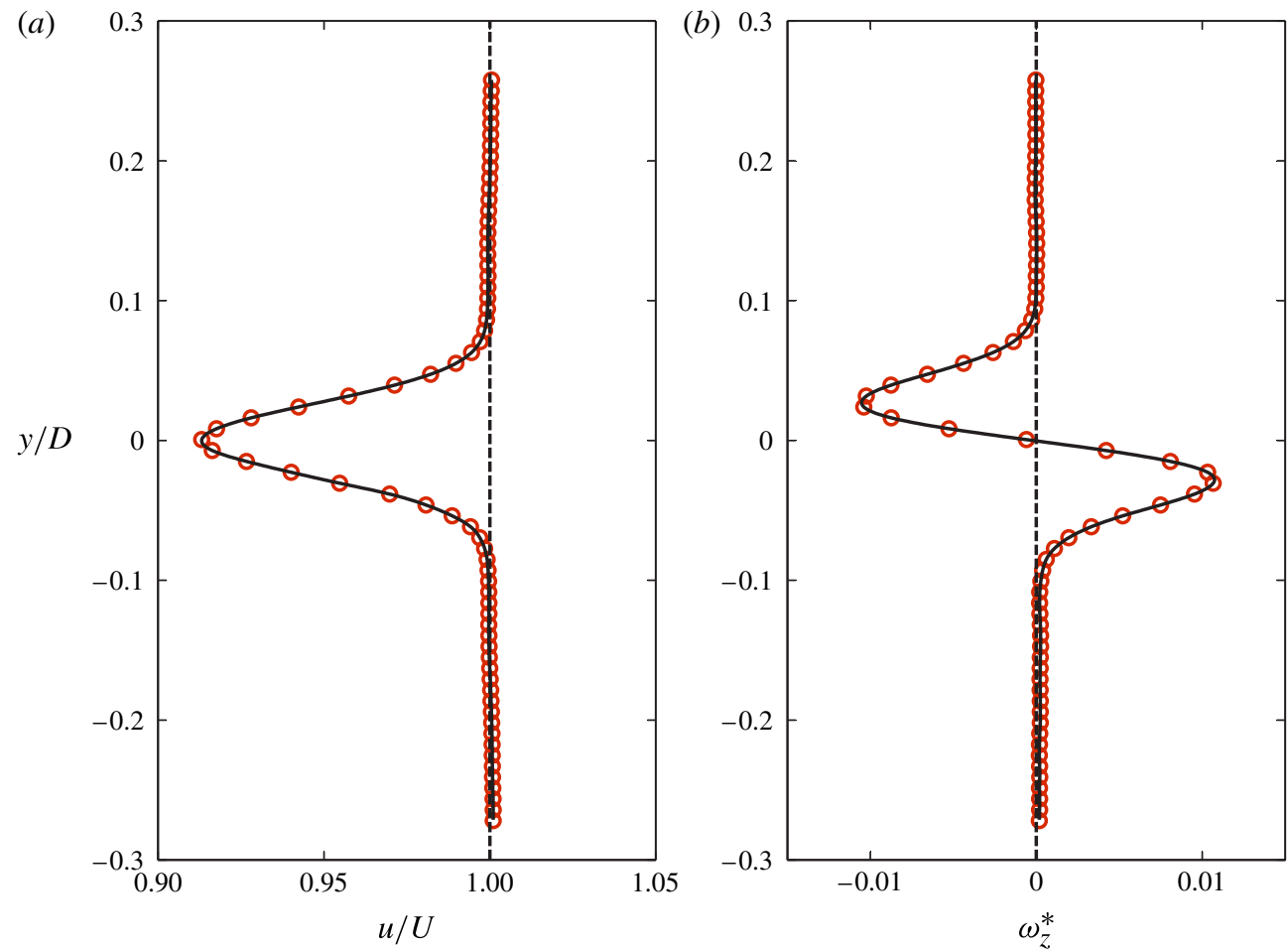

(c)

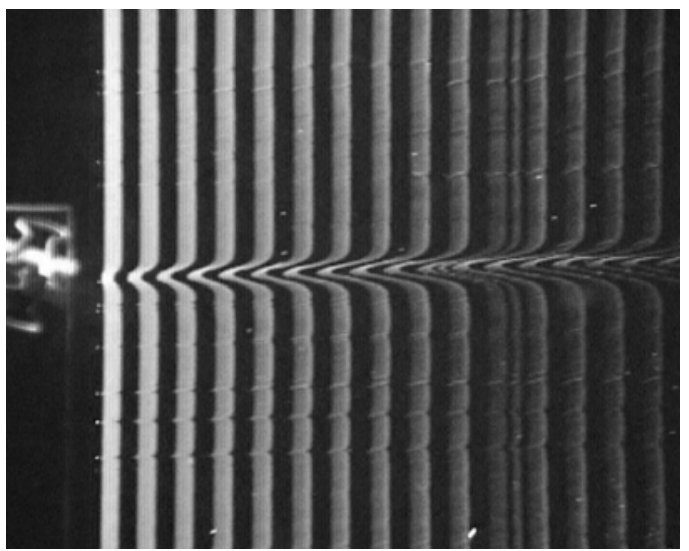

(d)

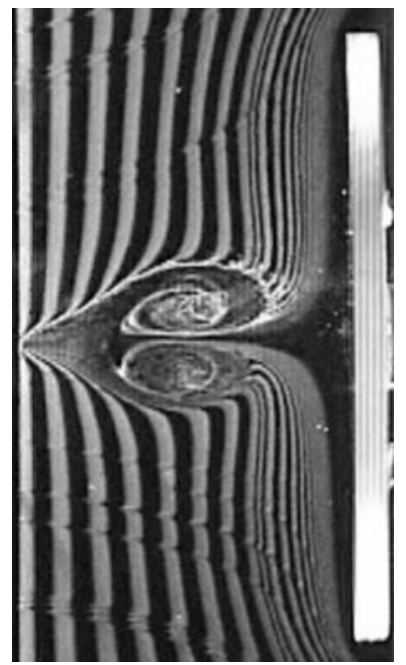

FIGURE 4. (Colour online) PIV measurements of the fishing-line wake at $x / D=0$ without a circular plate, showing the time-averaged velocity profile $(a)$ and the time-averaged spanwise vorticity profile $(b)$. Flow visualization pictures, showing the fishing-line wake $(c)$ and the development of a vortical structure after the impingement of fishing-line wake on a circular plate $(d)$.

end of vortex development was affected by the vortex shedding around the edge of the circular plate. Indeed, no causal relationship between the vortex shedding from the 

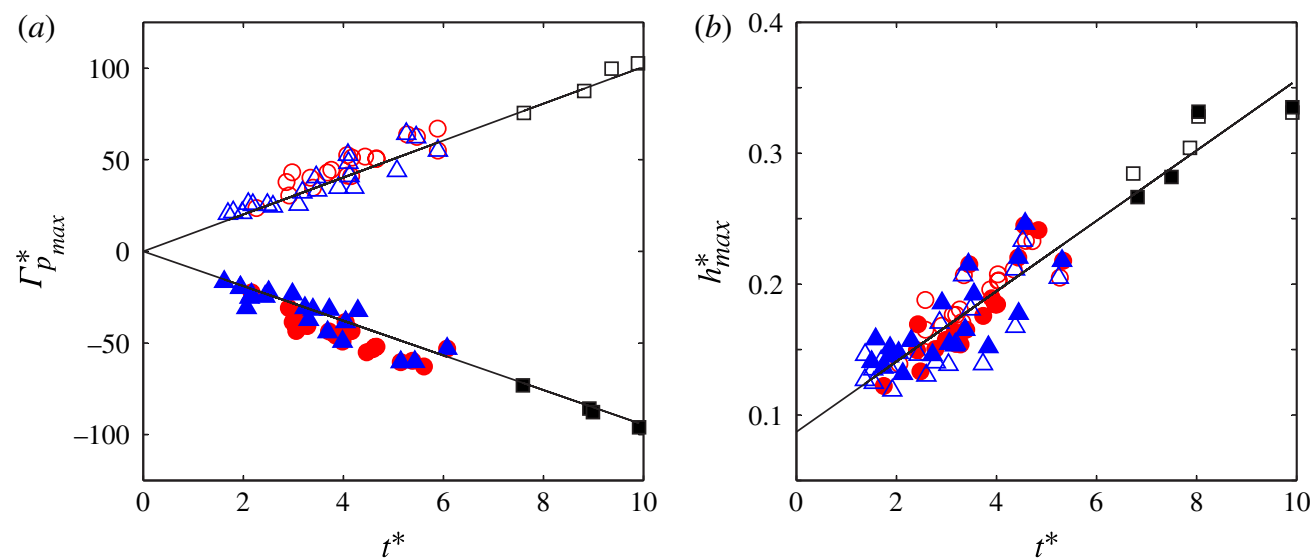

FIGURE 5. (Colour online) Variation in $(a)$ maximum circulation and $(b)$ maximum wallnormal distance of the primary vortices over a circular plate at the end of the linear growth: positive $(\bigcirc)$ and negative $(\bullet)$ type I-A vortices; positive $(\triangle)$ and negative $(\Delta)$ type I-B vortices; positive $(\square)$ and negative ( $\square$ ) type II vortices.

edge of the circular plate and the movement or the type of vortex development over the circular plate was observed from the flow visualization study.

Figure 5(a,b) show the variation of maximum circulation and maximum wall-normal displacement, respectively, in the vortex samples at the end of their linear growth. These figures suggest that the rate of vortex growth (i.e. the slope in each figure) is nearly identical among all samples regardless of the type of vortex development. Therefore, it makes sense to take a sample from each type to examine the behaviour of vortex development even if there are some variations in the quantitative values among the samples. Moreover, no differences between type I-A and type I-B developments are found in the figures (apart from a difference in the sign of circulation), supporting our claim that the mechanism of type I-A vortex development is essentially the same as that of type I-B development.

\subsection{Type I-A development}

Figure 6 gives a sequence of flow visualized pictures, showing a typical type I-A development of counter-rotating pair of vortices in the frontal stagnation region of a circular plate. A small vortex pair that is being formed in the centre of a circular plate is seen in the first picture (top left of figure 6). The subsequent vortex development is shown with an interval of $\Delta t^{*}=0.6$, changing a symmetric pair of circular vortices to an asymmetric elliptic configuration. This may suggest that the pair of counter-rotating vortices is undergoing elliptic instability, as the cross-section of the vortices became elliptical in shape due to their mutual strain field. Maximum vortex growth can be seen in the fourth picture (top right of figure 6) before the vortices are displaced in the positive $y$ direction.

Figure 7(a) shows an example of type I-A development based on PIV measurements, where the spanwise vorticity is non-dimensionalized by $\omega_{z}^{*}=\omega_{z} /\left(U / d_{0}\right)$. Note that PIV measurements were carried out separately from flow visualizations but under very similar conditions. Figure $7(b)$ shows that the non-dimensional circulation of primary vortices, $\Gamma_{p}^{*}=\Gamma_{p} / U d_{0}$, increases linearly with time until $h^{*}$ reaches its maximum value, suggesting that the vorticity flux from the laminar wake is constant during 

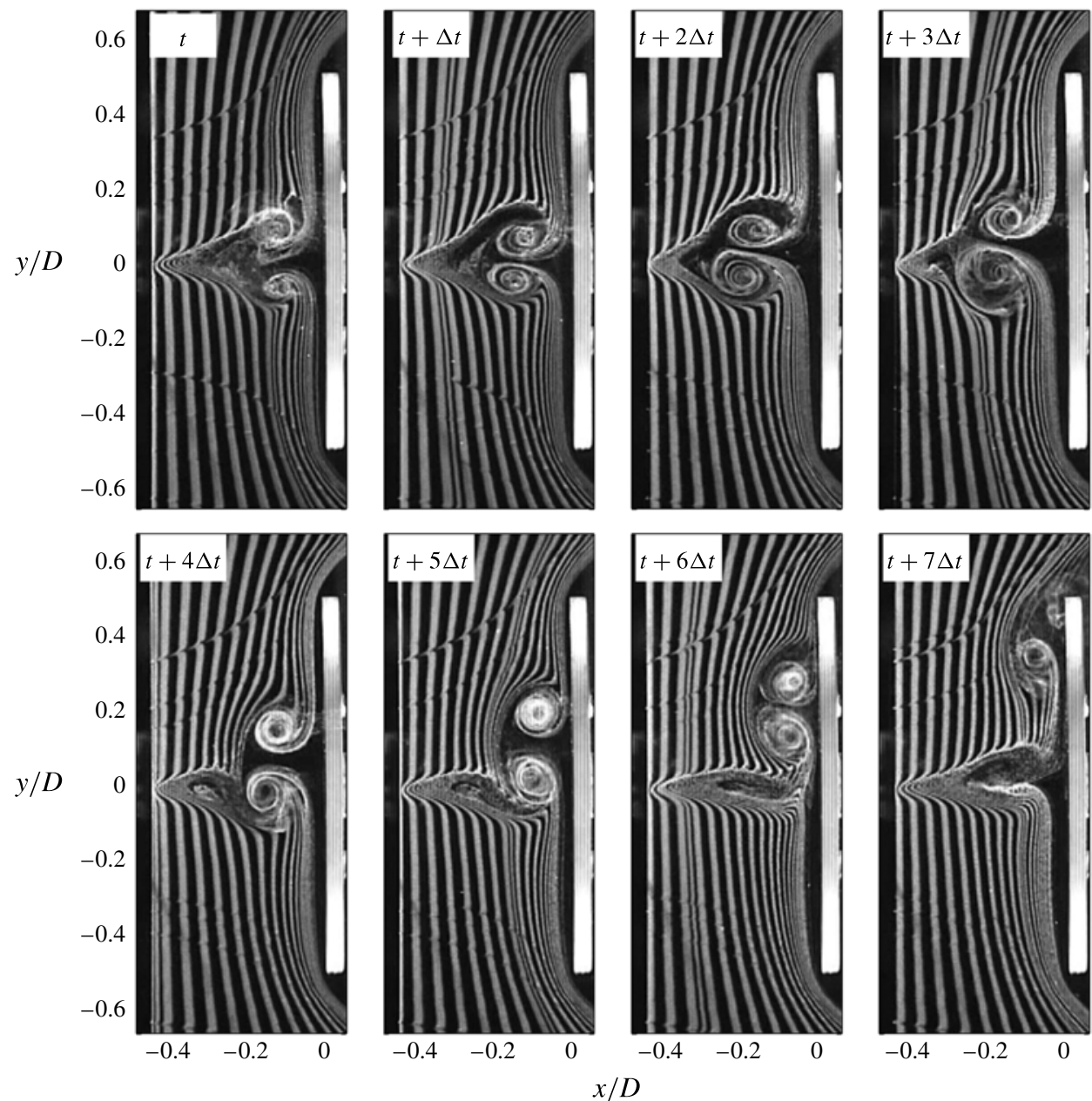

FIGURE 6. A sequence of flow visualized pictures to show type I-A development of a vortex pair in the stagnation region of a circular plate with a fine fishing line placed in the upstream. The time interval between the consecutive pictures is $\Delta t^{*}=0.6$.

this period. Here, $h^{*}$ is the non-dimensional distance of the vortices from the wall as defined by $h^{*}=h / D$. Unlike previous studies of counter-rotating vortex pairs in an open flow (Leweke \& Williamson 1998; Laporte \& Corjon 2000), the circulation of vortices in the present study does not remain constant. Non-dimensional time $t_{h_{\max }}^{*}$ and $t_{\Gamma_{\max }}^{*}$, defined as $t^{*}$ when $h^{*}$ and $\Gamma^{*}$, respectively, takes the maximum value, are indicated by the vertical lines in the figure. Although the rate of increase may be reduced after $t_{h_{\max }}^{*}=3.8$, the primary vortex circulation $\Gamma_{p}^{*}$ continues to increase until $t_{\Gamma_{\max }}^{*}=4.5$. As the primary vortices grow, the secondary vorticity $\Gamma_{s}^{*}=\Gamma_{s} / U d_{0}$ is induced in the near-wall region to maintain the non-slip condition at the wall. Figure $7(b)$ shows that the circulation of the secondary vorticity keeps pace with the increase in $\Gamma_{p}^{*}$, but the balance is broken at $t_{h_{\max }}^{*}=3.8$.

Figure $7(c)$ shows that the vortex diameter $d^{*}$ increases with $\sqrt{t^{*}}$ (or the crosssectional area of vortex increases linearly with time) to a maximum value of $d^{*}=0.17$ 

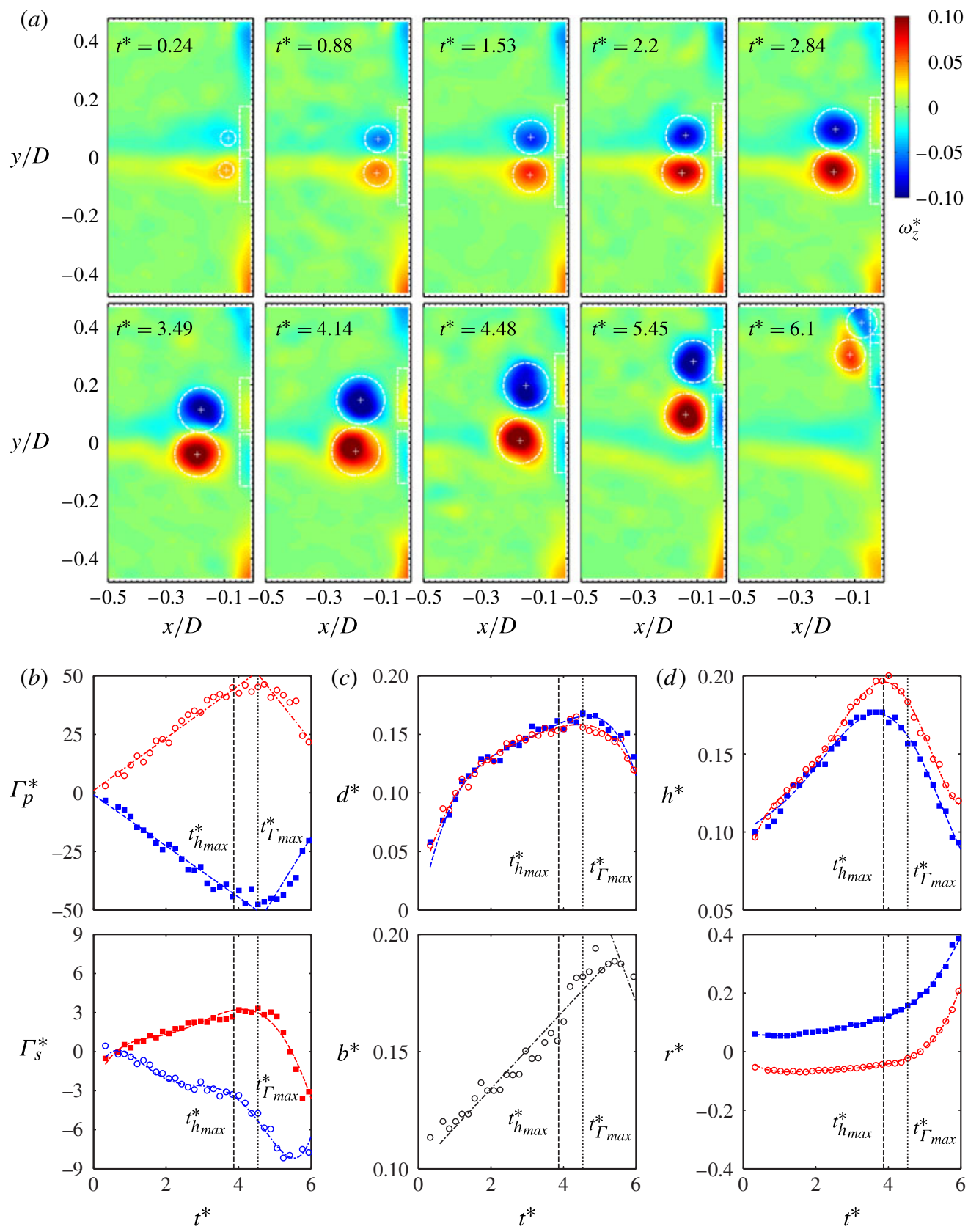

FIgURE 7. (Colour online) Type I-A development of a vortex pair in the stagnation region of a circular plate based on PIV measurements: $(a)$ changes in vorticity distribution in each vortex; $(b)$ changes in primary and secondary circulations; $(c)$ changes in vortex diameter and the core spacing; $(d)$ wall-normal and wall-parallel movement of the vortices.

at $t_{\Gamma_{\max }}^{*}=4.5$, confirming that the vorticity flux from the fishing-line wake is constant. It is also shown that the non-dimensional spacing between the vortex cores, $b^{*}=b / D$ increases linearly with time to a maximum value of $b^{*}=0.19$ at $t^{*}=5.2$. The vortex 
diameter to spacing ratio, $d^{*} / b^{*}$ takes a value of 0.90 at the end of type I-A growth. Figure $7(d)$ shows that the distance of a pair of vortices from the wall, $h^{*}$ increases linearly with time, indicating that the speed of the vortices moving away from the wall is constant at $\mathrm{d} h^{*} / \mathrm{d} t^{*}=0.02$. The vortex pair then starts to move off the plate centre, as indicated by the increase in spanwise spacing of these vortices, $r^{*}=y / D$ in figure $7(d)$.

As a pair of counter-rotating vortices starts moving off the centre of a circular plate at the end of vortex growth, the negative (shown in blue online) shear layer of the fishing-line wake no longer feeds on the negative (shown in blue online) primary vortex to increase its circulation. Instead, it starts to feed on the positive (shown in red online) primary vortex (see figure $7(a)$ at $t^{*}=4.8$ ). This is because the $y$ position of counter-rotating vortices relative to the fishing-line wake is shifted as a result of the antisymmetric distortion of vortices similar to that of elliptic instability (Leweke \& Williamson 1998; Laporte \& Corjon 2000). This can be seen in the flow visualization sequence, figure 6 , as well as in PIV results, figure 7(a). Therefore, the positive (shown in red online) primary vortex reduces its strength. At the same time, the circulation of the negative (shown in blue online) secondary vorticity increases, helping the pair of vortices move in the positive $y$ direction by induction. This reflects in a reduction of the rate of increase in $\Gamma_{p}^{*}$ as well as the shift of balance in $\Gamma_{s}^{*}$ between the positive (shown in red online) and negative (shown in blue online) secondary vortices at $t_{h_{\max }}^{*}=3.8$, as shown in figure $7(b)$. It should be noted that the leading vortex of a pair of vortices is always the one closer to the wall, which is the negative (shown in blue online) vortex in type I-A development. The wall-parallel displacement of the pair of vortices from the centre of a circular plate seems to be accelerated as the wall-normal antisymmetry is increased, as shown in figure $7(d)$. The antisymmetric deformation of counter-rotating vortices in the wall-normal direction coupled with the symmetric deformation in the wall-parallel direction suggests that the vortices may be undergoing short-wave elliptic instability (Leweke \& Williamson 1998).

\subsection{Type I-B development}

The mechanism of type I-B development is essentially the same as that of type I-A development, except that the direction of vortex movement along the circular plate in the late stage is opposite. In other words, type I-B development is statistically symmetric to type I-A development.

An example of type I-B development of a pair of vortices in the stagnation region of a circular plate is shown in figure 8 by a sequence of flow visualization pictures with an interval of $\Delta t^{*}=1.0$. The corresponding vorticity field based on PIV measurements is given in figure $9(a)$. Figure $9(b)$ shows a linear growth in circulation $\Gamma_{p}^{*}$ of a vortex pair until $t_{h_{\max }}^{*}=2.9$. This figure also demonstrates that there is a balanced growth between the positive (shown in red online) and negative (shown in blue online) secondary vorticity regions as a result of induction by the primary vortices. However, this balance is broken at $t_{h_{\max }}^{*}=2.9$ when the circulation of negative (shown in blue online) secondary vorticity $\Gamma_{s}^{*}$ starts to increase. At this point, the primary vortex pair starts to move towards the bottom edge of the plate (in the negative $y$ direction), as indicated by the reduction in $r^{*}$, figure $9(d)$. Similar to type I-A development, $d^{*}$ increases with $\sqrt{t^{*}}$ until $t_{h_{\max }^{*}}$ while $b^{*}$ increases linearly with $t^{*}$ as shown in figure $9(c)$. Eventually they reach to the maximum values of $d^{*}=0.14$ and $b^{*}=0.17$ at the end of type I-B growth, giving the vortex diameter to spacing ratio, 

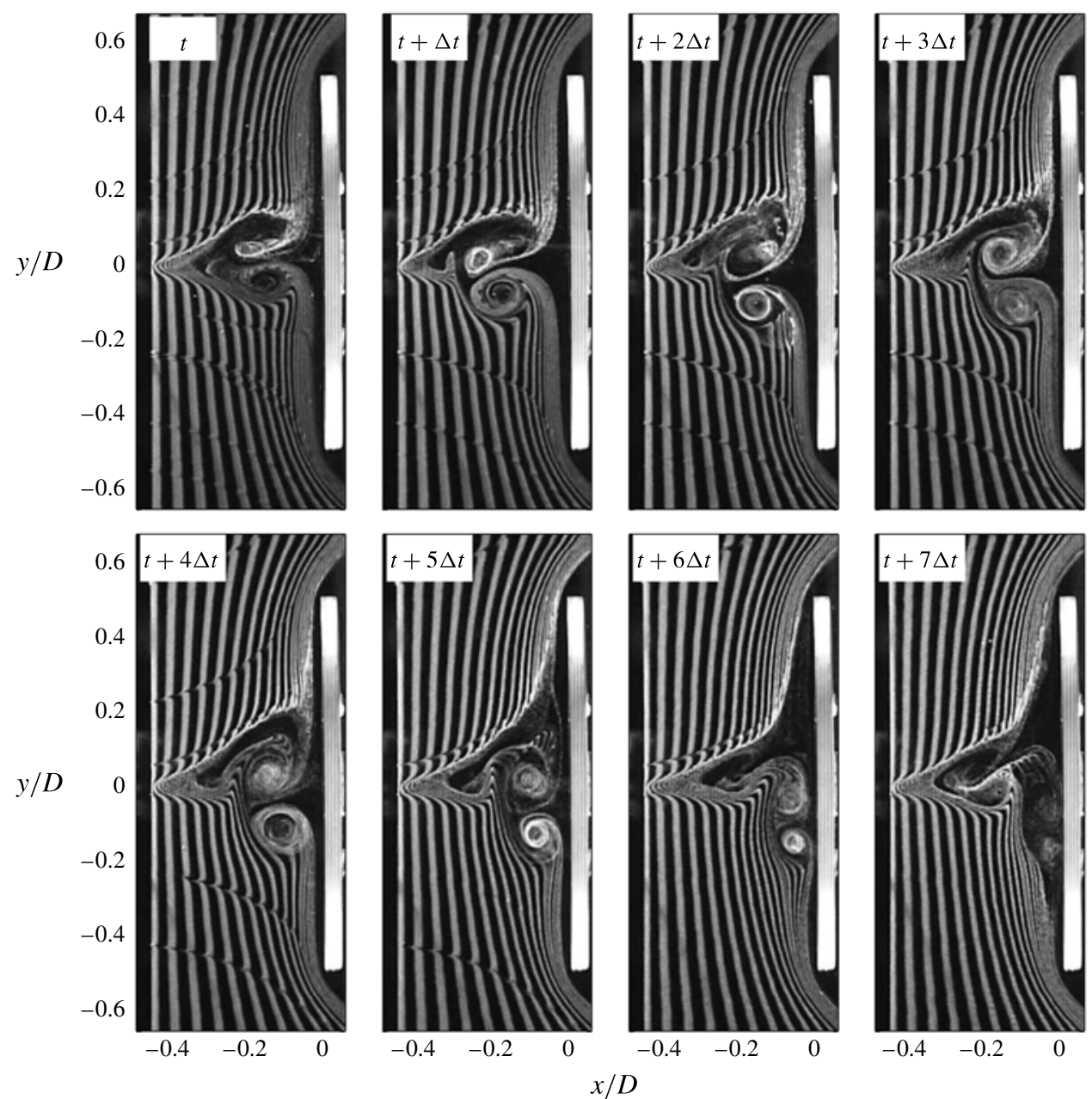

FIGURE 8. A sequence of flow visualized pictures to show type I-B development of a vortex pair in the stagnation region of a circular plate with a fine fishing line placed in upstream. The time interval between the consecutive pictures is $\Delta t^{*}=1.0$.

$d^{*} / b^{*}=0.82$. Moreover, the growth of $h^{*}$ is linear with time until $t_{h_{\max }}^{*}$, as shown in figure $9(d)$.

\subsection{Type II development}

Figure 10 shows an example of Type II vortical development over a circular plate in a flow visualization sequence. It can be seen that a pair of vortices is already developed in the first picture (top left of figure 10). The change of vortices from circular to elliptical in shape is clearly seen in this sequence of pictures, where the vortical interaction lasts for over $\Delta t^{*}=4.2$. The second picture from the bottom left in figure 10 is very similar to figure 9 of Leweke \& Williamson (1998), suggesting that the pair of counter-rotating vortices may be going through a short-wavelength elliptic instability. The next picture (third from the bottom left) shows the vortices in an early stage of breakdown, where the bottom part of the fluid in the negative (top) vortex is drawn to the positive (bottom) vortex and vice versa. This process of fluid 

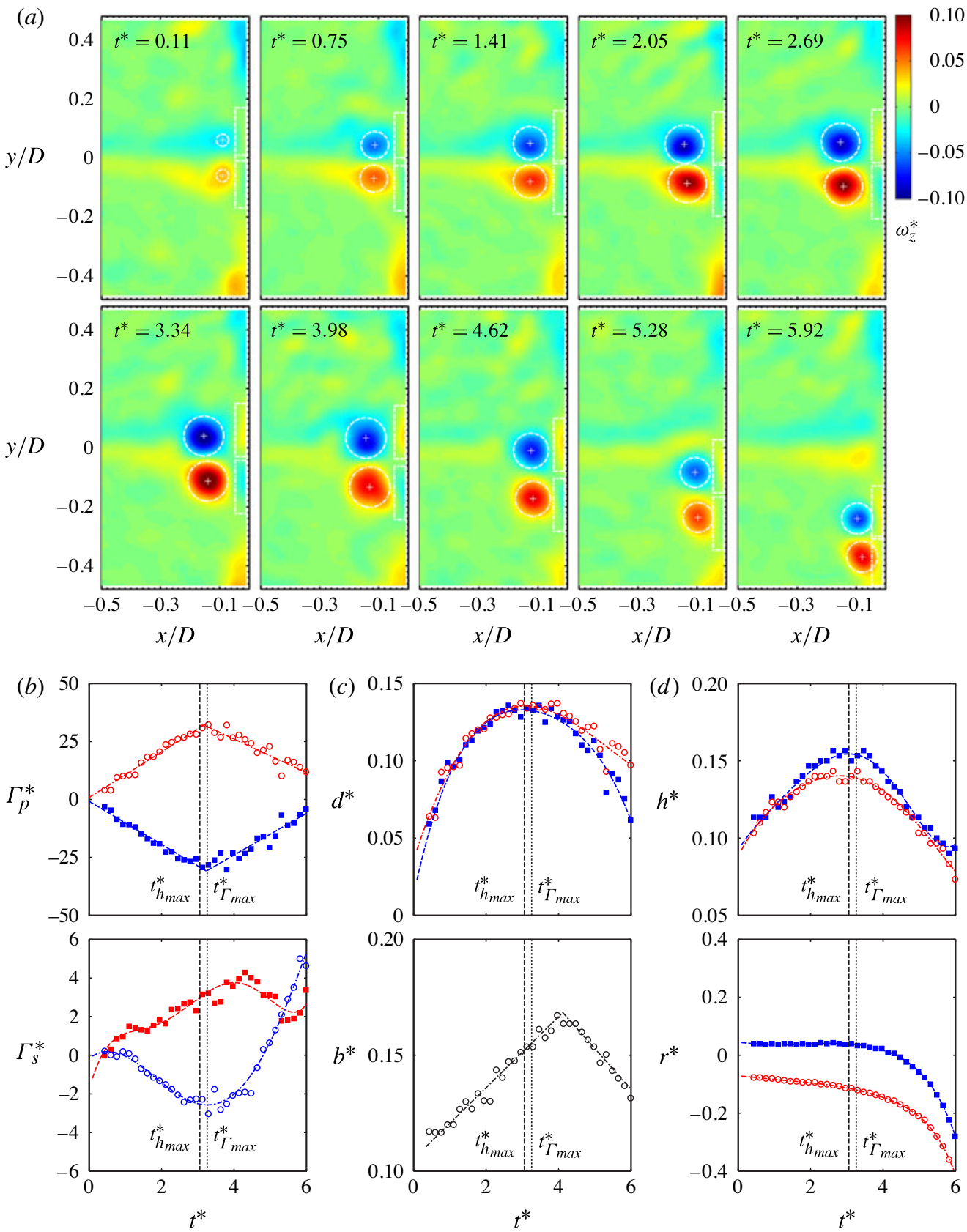

FIgURE 9. (Colour online) Type I-B development of a vortex pair in the stagnation region of a circular plate based on PIV measurements: $(a)$ changes in vorticity distribution in each vortex; $(b)$ changes in primary and secondary circulations; $(c)$ changes in vortex diameter and the core spacing; $(d)$ wall-normal and wall-parallel movement of the vortices.

mixing initiates a vortex breakdown as shown in the last picture (bottom right), and the residual vorticity moves away from the centre of the circular plate to the negative $y$ direction (bottom of the picture). 

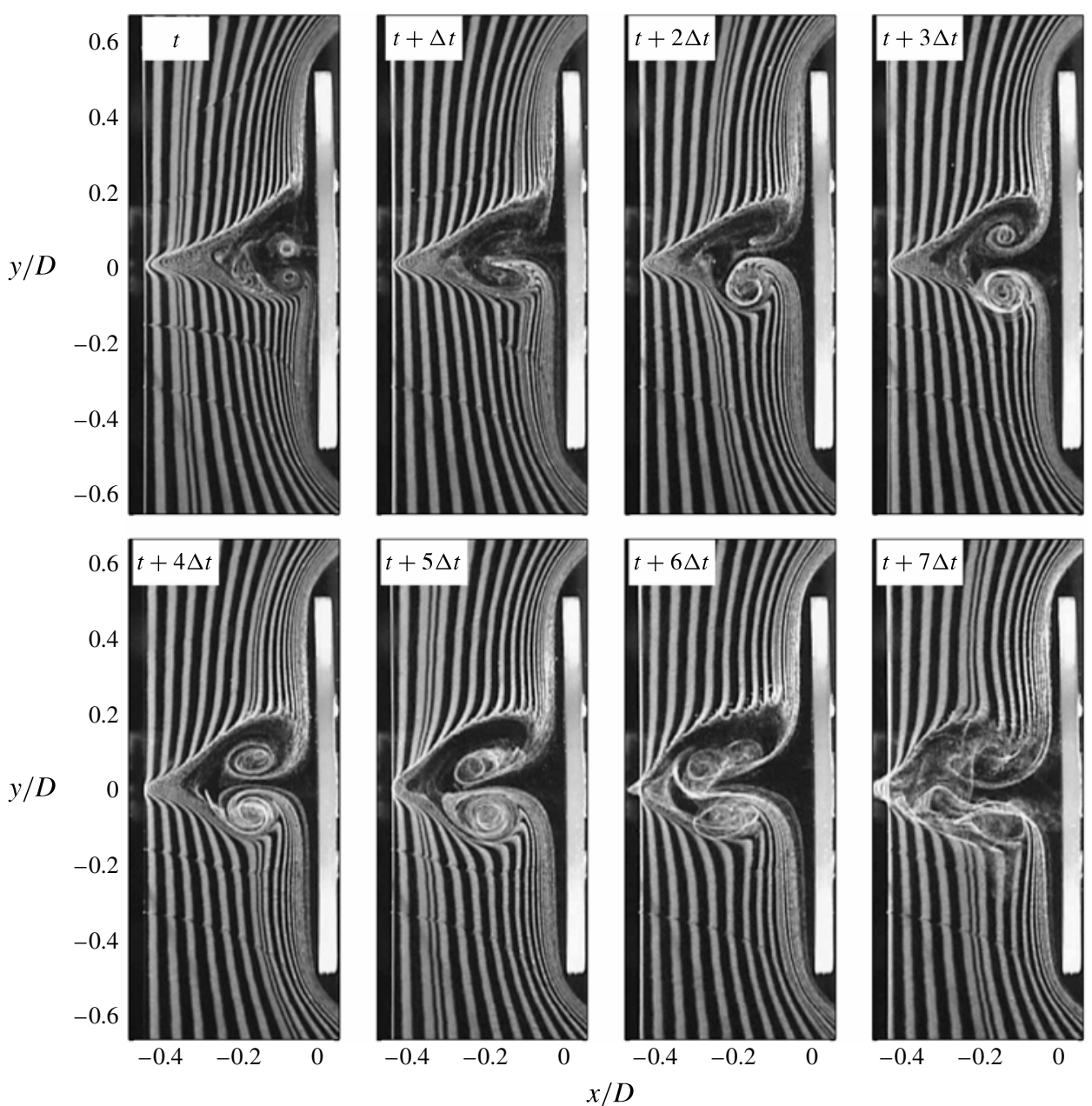

FIGURE 10. A sequence of flow visualized pictures to show type II development of a vortex pair in the stagnation region of a circular plate with a fine fishing line placed in upstream. The time interval between the consecutive pictures is $\Delta t^{*}=0.6$.

Type II development of vortices based on PIV measurements is shown in figure $11(a)$. Figure 11(b) indicates that the growth of the primary vortex circulation $\Gamma_{p}^{*}$ in type II development lasts more than twice as long as in type I. The vortices grow in size as they move away from the wall until they reach the maximum value of $h^{*}=0.35$ at $t_{h_{\max }}^{*}=8$. However, the circulation of secondary vorticity region $\Gamma_{s}^{*}$ remains nearly constant despite of the linear growth in $\Gamma_{p}^{*}$. This is because the primary vortices detach from the wall much faster than the vortex growth. In other words, the vortices are in the detached amplifying regime (Bae et al. 2000). The vortex diameter takes a maximum value of $d^{*}=0.23$ when the vortex core spacing increases to $b^{*}=0.25$ at $t^{*}=10$ as shown in figure $11(c)$. This gives a diameter-to-spacing ratio of $d^{*} / b^{*}=0.92$, which is slightly greater than that in type I. The vortices stay in the stagnation region of the circular plate before moving off the centre towards the negative $y$ direction in this example. 

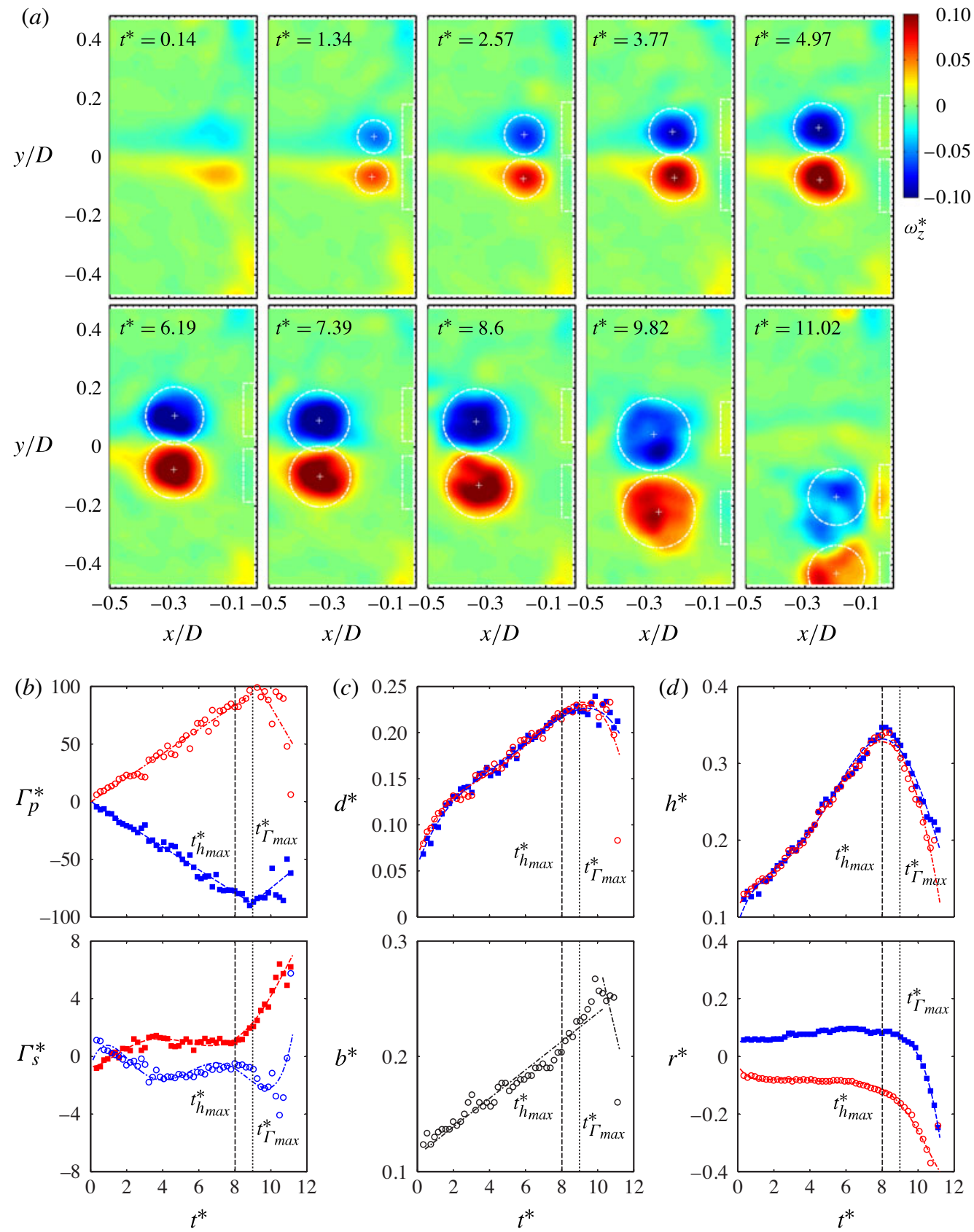

FIgURE 11. (Colour online) Type II development of a vortex pair in the stagnation region of a circular plate based on PIV measurements: $(a)$ changes in vorticity distribution in each vortex; $(b)$ changes in primary and secondary circulations; $(c)$ changes in vortex diameter and the core spacing; $(d)$ wall-normal and wall-parallel movement of the vortices.

\section{Vorticity amplification}

As the wake of a fishing line impinges on the wall, the vorticity in wake shear layer is steadily deposited in the centre of a circular plate. However, this deposition of 
vorticity in the stagnation region of the circular plate does not guarantee the creation or growth of vortices. It is the stretching of vorticity by the mean strain of diverging flow (Sutera 1965; Sadeh \& Brauer 1980) around the circular plate that is necessary for the formation and growth of vortices in the stagnation region.

To verify this mechanism, we have carried out another flow visualization experiment using the hydrogen bubble technique, where a fishing line was placed in the upstream of a rectangular plate, $60 \mathrm{~mm}$ wide (same as the diameter of a circular plate in the present study) and $400 \mathrm{~mm}$ long, along its length. Therefore, the boundary-layer growth along the width of this plate is the same as that over the circular plate. The result shows that the vorticity of the fishing-line wake was accumulated in the stagnation region, growing to a size comparable to the vortices over the circular plate. However, no vortex roll-ups were observed. This is because the mean strain rate along the length of the rectangular plate is only $15 \%$ of that of the circular plate, therefore the vortex stretching along the length of the rectangular plate is much less than that around the circular plate. This is why the accumulated vorticity did not roll up into vortices. This observation supports the idea that the stretching of vorticity is crucial for the formation and growth of vortices in the stagnation region (Sutera 1965; Sadeh \& Brauer 1980).

The size of flow disturbance and the boundary-layer thickness over a bluff body also play an important role in an early stage of vortical development in the stagnation region. For example, Xiong \& Lele (2004) concluded that the ratio of disturbance length scale $l_{d}$ to the Hiemenz boundary-layer thickness $\delta$ is a crucial parameter to determine the amplification of vorticity in the free stream. The maximum amplification of disturbances occurred when the length-scale ratio, $l_{d} / \delta$ is about two. Similar results were also obtained in other studies (Kestin \& Wood 1970; Dhanak \& Stuart 1995; Bae et al. 2000)).

In the present study, the thickness of the Homann boundary layer (Homann 1936) over a circular plate was obtained from the PIV measurement, to give $\delta \approx 1.8 \mathrm{~mm}$ (see figure 2). For the disturbance length scale, the wake half-width of a fishing line was measured to give $l_{d} \approx 3.6 \mathrm{~mm}$ (see figure 3 ). These values, therefore, give the length-scale ratio between the size of flow disturbance (the wake half-width of a fishing line) and the boundary-layer thickness of $l_{d} / \delta \approx 2$, agreeing very well with the optimum length-scale ratio obtained by Kestin \& Wood (1970), Dhanak \& Stuart (1995), Bae et al. (2000) and Xiong \& Lele (2004).

These results are consistent with previous studies on the formation of vortices and their initial growth. However, a further growth of vortices requires an accumulation of shear-layer vorticity deposited by the fishing-line wake. Our results indicated that the vortices in type II development increase their size by nearly twice those in type I (see figure $11 c$ ). As a result, the length-scale ratio increases by the same amount. This shifts these vortices from attached to detached amplifying regime (Bae et al. 2000), suggesting that the stagnation-point heat transfer may be reduced in type II vortical development.

\section{Development of vortical instability}

The side view of a vortex pair in the late stage of type I development over a circular plate is depicted in figure 12 by flow visualization using the hydrogenbubble technique. Here, the viewing angle is rotated by $90^{\circ}$ from the previous cases (figure 5-11) through the central axis of a circular plate. Two hydrogen-bubble wires are placed parallel to each other in an area where the vortex pair is formed 
(a)

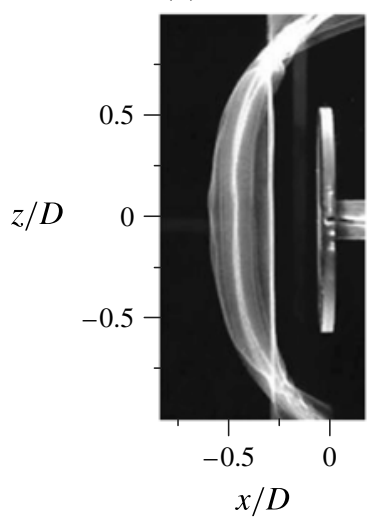

(b)

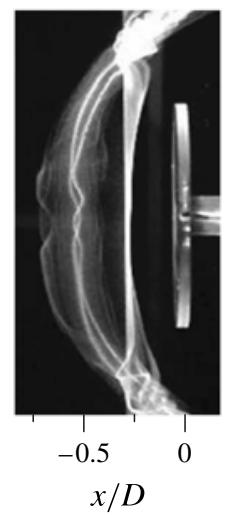

(c)

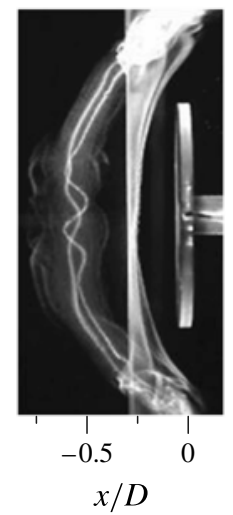

$(d)$

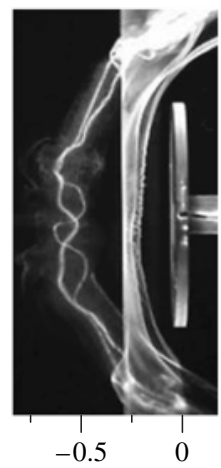

$x / D$ $(e)$

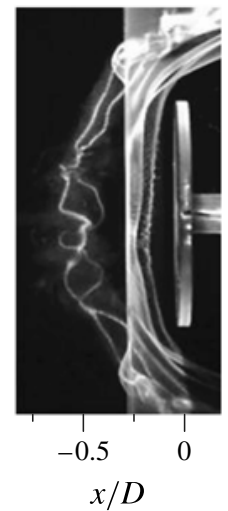

FIGURE 12. Side view of a pair of vortices over a circular plate showing elliptic instability at $t^{*}=3.0(a), 3.4(b), 3.6(c), 3.8(d)$ and $4.0(e)$, where $t^{*}$ is the non-dimensional time from the start of vortex formation. Two hydrogen-bubble wires were placed in the area where the vortex pair is formed, thereby vortex filaments can be visualized as a result of the concentration of hydrogen bubbles in the vortex cores.

(at $x / D=-0.25$ and $y / D= \pm 0.12$ ), thereby vortex filaments can be visualized by the concentration of hydrogen bubbles in the vortex cores. However, one of the hydrogen-bubble wires cannot be seen in the figure since it is immediately behind the other.

The rate of growth of the two vortices is nearly identical until $t^{*}=3.0$, where $t^{*}$ is the non-dimensional time from the start of vortex formation. At $t^{*}=3.4$ (see figure 12), the vortices start to show an antisymmetric waviness along the vortex filaments similar to that observed in the short-wavelength elliptic instability (Leweke \& Williamson 1998), growing its amplitude until breakdown at $t^{*}=4.0$. During this period of instability growth, the non-dimensional wave amplitude, $d_{c}^{*}=d_{c} / D$ increases from 0.06 to 0.19 , while the non-dimensional wavelength $\lambda^{*}=\lambda / D$ changes from 0.29 to 0.38 . This suggests that in this particular case the wavelength-to-waveamplitude ratio, $\lambda^{*} / d_{c}^{*}$ reduces from 4.8 to 2.0 within the period of $\Delta t^{*}=0.8$. The non-dimensional vortex diameter, $d^{*}=d / D$ observed in late stage of type I vortex development typically takes a value between 0.17 and 0.22 , therefore the wavelength to vortex diameter ratio is $\lambda^{*} / d^{*}=1.7-2.2$.

The antisymmetric deformation of vortex cores observed over a circular plate in the present study has a remarkable resemblance to that shown by Leweke \& Williamson (1998), who generated the vortices in a water tank by squeezing two parallel plates. For example, the flow visualization picture shown in figure 12 has a strong similarity to figure $5(b)$ of Leweke \& Williamson (1998), where a nearly identical value of $\lambda^{*} / d^{*}=2.0 \pm 0.2$ was obtained for the wavelength-to-vortex-diameter ratio. However, the present vortex core spacing is less than that of Leweke \& Williamson (1998) and Laporte \& Corjon (2000). The core-spacing-to-vortex-diameter ratio in the present test is $b^{*} / d^{*} \approx 1.0$, which gives the wavelength-to-core-spacing ratio $\lambda^{*} / b^{*}=1.7-2.2$. In contrast, the corresponding values in an unforced system of Leweke \& Williamson (1998) are $b^{*} / d^{*} \approx 2.5$ and $\lambda^{*} / b^{*}=0.77 \pm 0.02$.

Flow visualization pictures in figures 6,8 and 10 demonstrate that the streak lines around the vortex cores become elliptical in shape during the vortical 

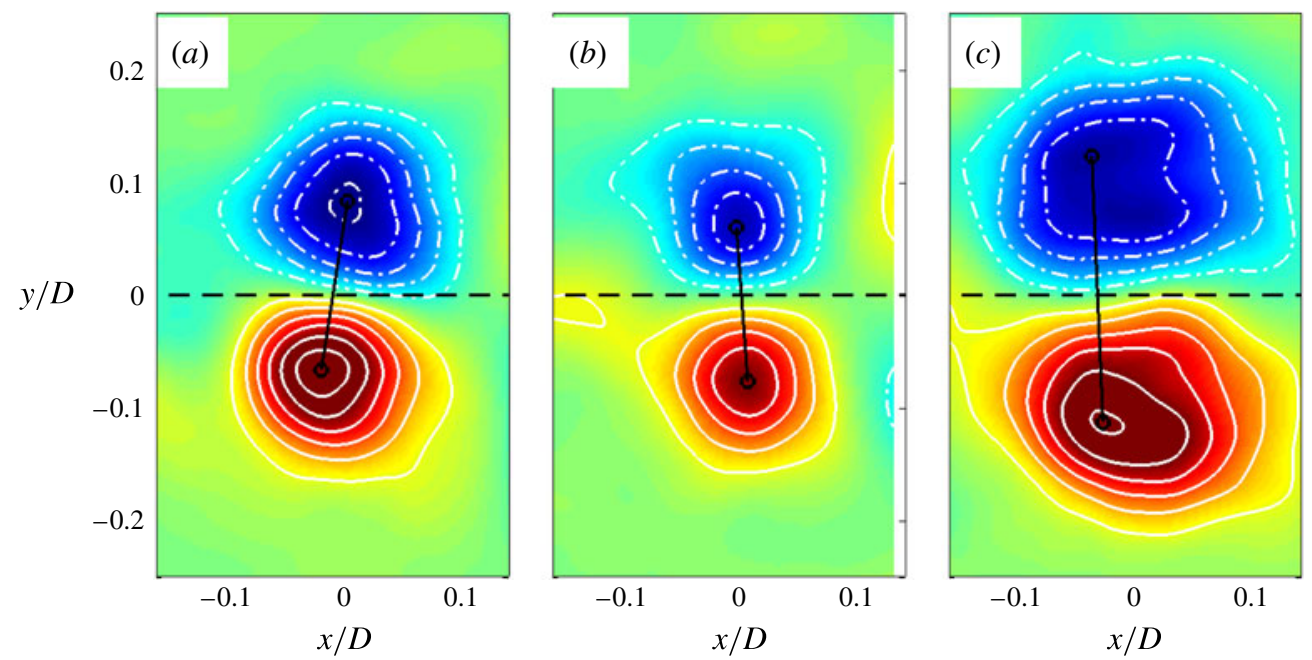

FIGURE 13. (Colour online) Vorticity contours of a pair of the vortices at the end of linear circulation growth, where $x / D=0$ corresponds to the mean wall-normal distance of vortical cores: (a) type I-A development; $(b)$ type I-B development; $(c)$ type II development.

development, suggesting that this deformation may be caused by the elliptic instability. Figure 13(a) also shows an elliptic-shaped contour of the non-dimensional vorticity $\omega_{z}^{*}=\omega_{z} /\left(U / d_{0}\right)$ of a pair of vortices during type I-A development when the wallnormal displacement $h^{*}$ of the vortices becomes maximum. Here, the difference in wall-normal displacement between the two vortices is greatest at $t_{h_{\max }}^{*}=3.8$, yet the pair still remains in the centre of a circular plate. The corresponding vorticity contour of a vortex pair during type I-B development is shown in figure 13(b), again showing an elliptic deformation of vorticity contour at $t_{h_{\max }}^{*}=2.9$. A further similarity to the elliptic instability can be seen in figure 13(c), showing a large elliptical deformation of vortex cores during type II development.

The cores of counter-rotating vortices deform in a cooperative manner during the short-wavelength elliptic instability, in such a way that the wall-parallel deformation is symmetric while they become antisymmetric in the wall-normal direction (Leweke \& Williamson 1998). A similar vortical deformation is illustrated in the schematic view of figure 14 adopted from Leweke \& Williamson (1998). Here, a pair of vortices is shown in the plan view (top) and the side view (bottom), where the vortices have positive (shown in red online) and negative (shown in blue online) vorticity, respectively. A vertical plane (shown in green online) through the centre of a circular plate indicates a laser light sheet used for flow visualization and PIV measurements in the present study. Figure 14(a) shows a pair of vortices during type I-A development, where a positive (shown in red online) vortex moves further away from the wall than a negative (shown in blue online) vortex in the centre of the plate. At the same time, the vortices move together towards the top edge of a circular plate, where the leading vortex is the one closer to the wall: the negative (shown in blue online) vortex in this case. The vortex cores are seen to deform in a similar cooperative manner in the final vortical development of type I-B, as shown in figure 14(b). Here, a symmetric wall-parallel deformation of a pair of vortices is led by a positive (shown in red online) vortex, which stays closer to the wall during the wall-normal deformation. 
(a)
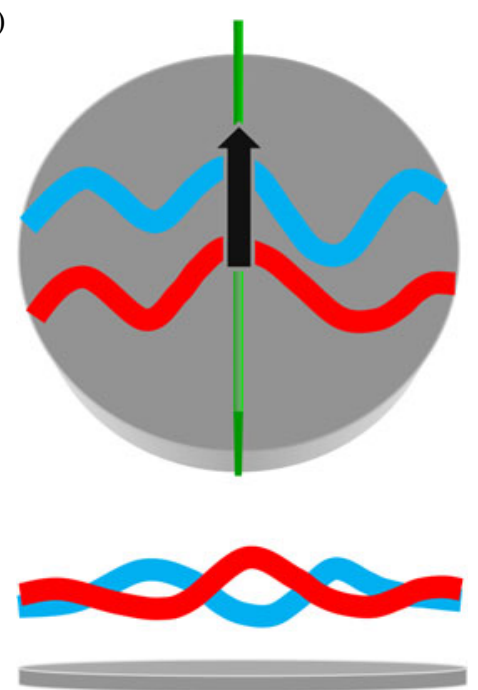

(b)
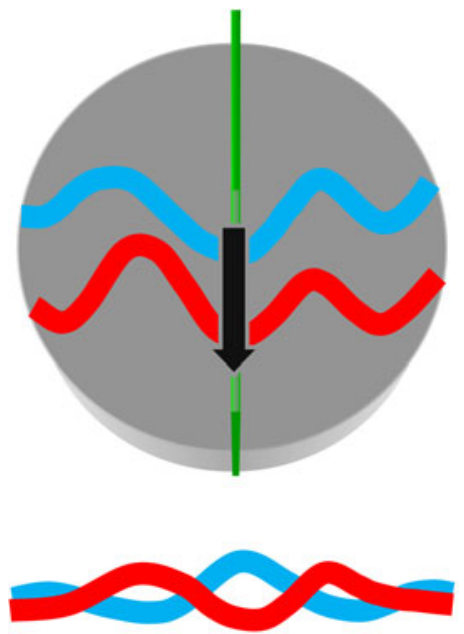

FIgURE 14. (Colour online) Schematics of vortex filaments during elliptic instability, adopted from Leweke \& Williamson (1998). The plan view (top) shows a symmetric deformation of vortices parallel to the wall, while the side view (bottom) shows an antisymmetric vortical deformation in wall-normal direction: $(a)$ type I-A development; $(b)$ type I-B development.

As demonstrated above, there are similarities between the present vortical instability and that of Leweke \& Williamson (1998) which would suggest that the vortical instability observed in our experiment may be due to the elliptic instability. We should note, however, that there are also differences in the experimental setting to suggest otherwise. For example, the flow is unforced in Leweke \& Williamson (1998), where the elliptic instability develops due to the weak strain from the mutual induction of the cooperative vortices. In the present study, on the other hand, there is a strong external strain around the stagnation point (see figure 3). Also, the elliptic instability in Leweke \& Williamson (1998) develops along essentially a pair of straight vortices, whereas a significant curvature exists along the vortex core in the present study (see figure 12).

\section{Concluding remarks}

The formation, growth and instability of a pair of counter-rotating vortices over a circular plate in the downstream of a thin fishing line have been investigated using PIV and flow visualization techniques. Initially, the vortex pair in an axisymmetric stagnation flow was small, but it grew steadily by accumulating the shear-layer vorticity of the wake before going through vortical instability. We identified two types of vortical development, type I and type II. Nearly all vortical developments were type I, which was subdivided into type I-A and type I-B depending on the direction of wall-parallel movement of a vortex pair in the late stage. Type II development of a vortex pair, on the other hand, was a rare case, which was observed only in less than $5 \%$ of the total.

The circulation of the vortex pair in type I development increased linearly with time, reflecting a constant flux of vorticity impinging on the plate wall. As a result, the cross-sectional area of the vortices and the spacing between the vortex pair increased linearly with time. The distance of the pair of vortices from the wall also increased 
linearly with time, indicating that the wall-normal speed of the vortices was constant during the vortex growth. Towards the end of growth, the pair of vortices went through an antisymmetric deformation of vortex cores in the wall-normal direction while the wall-parallel deformation was symmetric. Later, the vortices moved away together from the central stagnation region towards the edge of the circular plate. Here, the leading vortex was always closer to the wall. The vortices stayed in the stagnation region much longer during type II development, increasing the vortex diameter nearly twice that of type I vortices. This initiated a breakdown of vortices before the residual vorticity moved away from the centre. The change of vortices from circular to elliptical in shape was clear in the results, suggesting that the pair of counter-rotating vortices may be going through a short-wavelength elliptic instability.

The present results were also consistent with previous results where both the vortex-stretching mechanism and the length-scale ratio were important factors in the formation of vortices and their growth in early stage of development. However, a further growth of vortices required an accumulation of shear-layer vorticity deposited by the fishing-line wake.

The antisymmetric deformation of vortex cores formed in an axisymmetric stagnation region over a circular plate had a remarkable similarity to that of shortwavelength elliptic instability of a counter-rotating vortex pair in an open system. In particular, the present flow visualization picture and the PIV results on mean streamlines within the vortex cores had a strong similarity to figure $5(b)$ of Leweke \& Williamson (1998), where a nearly identical value of wavelength to vortex diameter ratio was obtained. However, the present vortex core spacing was less than that of Leweke \& Williamson (1998) and Laporte \& Corjon (2000). It is considered that the disturbance due to vortical instability could be partially responsible for unexpectedly high heat transfer rate in the stagnation region of bluff bodies.

\section{Acknowledgements}

This investigation was supported by the National Natural Science Foundation of China under Grant Numbers 11002015 and 10832001 and by the Royal Academy of Engineering of the UK through the UK-China exchange programme. The authors would like to thank the referees for their valuable comments and suggestions that helped to improve the manuscript.

\section{Appendix. Vortex identification technique}

As a part of continuing effort to understand the development of turbulent boundary layers and their structures, a number of vortex identification techniques have been proposed over the years (Robinson 1991; Adrian 2007). In general, vortex identification techniques can be classified into two categories. The first category of the techniques aims at describing the pointwise swirling motion of vortices by invariants or eigenvalues associated with the velocity gradient tensor, e.g. the criteria based on $Q$, $\Delta, \lambda_{2}$ and $\Lambda_{c i}$. Here, $Q$ is the second invariant of the velocity gradient tensor (Hunt, Wray \& Moin 1988); $\Delta$ is the complex eigenvalue of the velocity gradient tensor (Chong, Perry \& Cantwell 1990); $\lambda_{2}$ is the second eigenvalue of the sum of squared symmetric part and squared antisymmetric part of the velocity gradient tensor (Jeong \& Hussain 1995); and $\Lambda_{c i}$ is the imaginary part of complex eigenvalue of the velocity gradient tensor (Zhou et al. 1999). Here, the spatial concentration of these values can be regarded as evidence of vortex existence. 

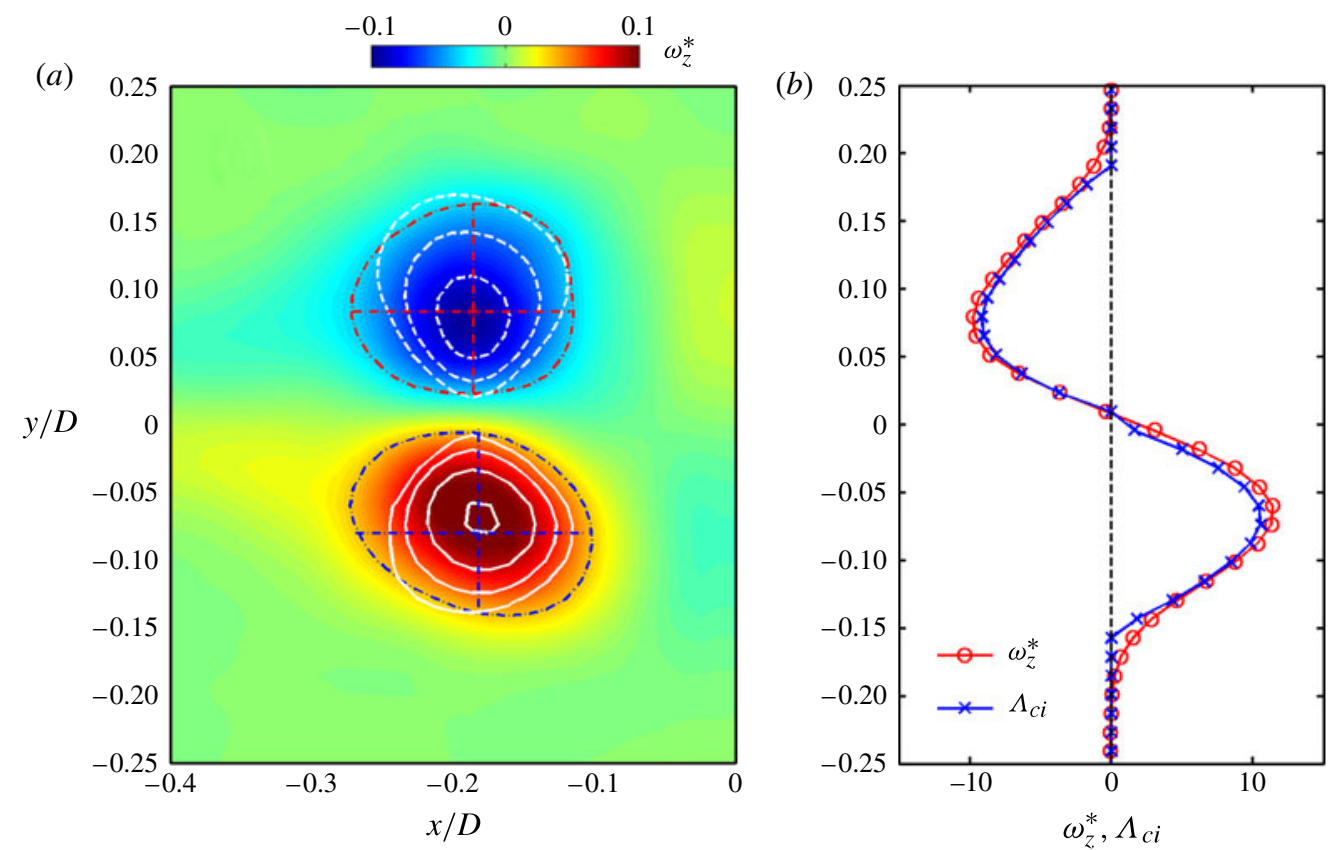

FIGURE 15. (Colour online) (a) Detection of vortices based on the spanwise vorticity $\omega_{z}^{*}$ (indicated by the colour level) within the stagnation flow over a circular plate, where the vortical centres are identified as a weighted geometrical centroid (dots, shown in red and blue online) of the threshold vorticity. This is compared with a technique based on $\Lambda_{c i}$ distribution (solid lines, positive; dashed lines, negative), where the vortex centres are given by the peak values in $\Lambda_{c i}$. (b) Distribution of $\omega_{z}^{*}$ and $\Lambda_{c i}$ at $x / D=-0.193$ showing a Gaussian-like distribution.

The second category of vortex identification techniques is based on pattern recognition. Usually, the velocity field containing vortices is scanned through with a template based on theoretical vortex models. The Oseen vortex is commonly used for this purpose, which has a circular shape with a Gaussian-like vorticity distribution. Two-dimensionality of the Oseen vortex makes it possible to apply this technique to the dataset of two-dimensional PIV. For example, Scarano, Benocci \& Riethmuller (1999) successfully applied this vortex identification technique to a PIV velocity field in order to deduce spanwise roller structures in a flow with backward facing step. Carlier \& Stanislas (2005) and Stanislas, Perret \& Foucaut (2008) obtained geometrical parameters of eddy structures by using a generalized Oseen vortex template in an incompressible turbulent boundary layer, while Pirozzoli, Bernardini \& Grasso (2008) applied a similar pattern reorganization technique to gain vortex core statistics in a supersonic turbulent boundary layer.

The present vortex detection technique, which has been described in the experimental set-up section, uses the spanwise vorticity as the detection criteria. The justification for this is as follows. First, the base flow over a circular plate is irrotational except for a very thin region close to the plate where a boundary layer is being developed. Second, the vorticity within the fishing-line wake is too small to affect the vorticity distribution within the core region of the stagnation vortex pair. Indeed, figure $3(b)$ demonstrates that the fishing-line wake has vorticity $\omega_{z}^{*}<0.01$, which is much less than the threshold value of $\omega_{z}^{*}=0.035$ used in the present study. 

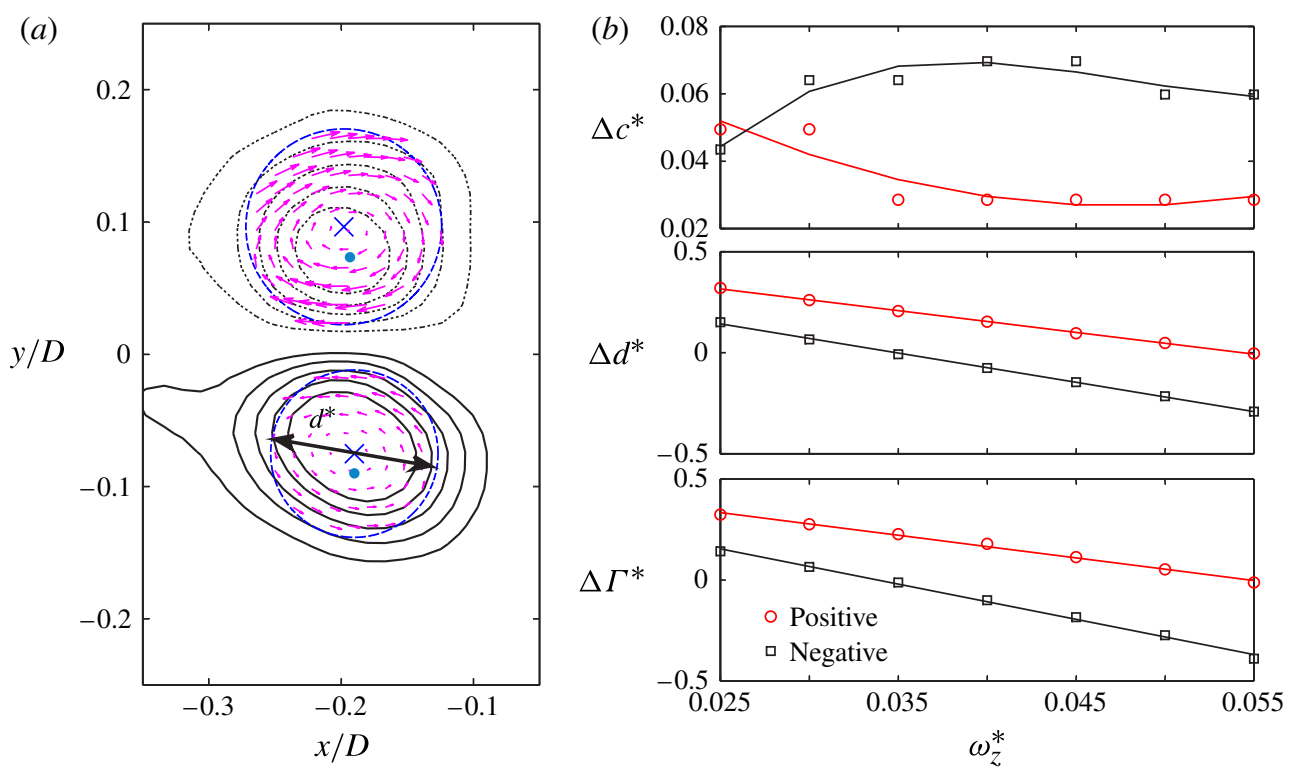

FIGURE 16. (Colour online) Detection of vortices by fitting the Oseen vortex profile. (a) Vortex periphery of the fitted vortices is indicated by blue chained lines, while the instantaneous velocity vectors are shown by red arrows in a reference frame moving with the vortices. Vorticity contours between $\omega_{z}^{*}=0.02$ and 0.08 are shown by black lines (solid, positive; dotted, negative) with an interval of $\Delta \omega_{z}{ }^{*}=0.015$. The crosses indicate the vortex centres by fitting the Oseen vortex profile, while the dots are the vortex centres determined by the present vortex identification technique with a vorticity threshold of $\omega_{z}^{*}=0.035$. (b) The relative difference in the vortex centre $\Delta \mathrm{c}^{*}$, vortex diameter $\Delta d^{*}$ and the circulation $\Delta \Gamma^{*}$ between the Oseen vortex fitting technique and the present technique as a function of the vorticity threshold $\omega_{z}^{*}$.

Finally, and most importantly, the distribution of vorticity $\omega_{z}^{*}$ in the vortex core region is very similar to that of the traditional swirl indicator, such as $\Lambda_{c i}$ (the imaginary part of complex eigenvalue of the velocity gradient tensor) in an axisymmetric stagnation flow. Figure 15 gives a comparison of the distributions of $\omega_{z}^{*}$ with $\Lambda_{c i}$, showing that the location of weighted geometrical centroid of the detected vortex (dots, shown in red and blue online) is very close to the peak in $\Lambda_{c i}$. Moreover, both $\omega_{z}^{*}$ and $\Lambda_{c i}$ seem to have a Gaussian-like distribution around the centroid, which is evident from figure $15(b)$.

We can also compare the present vortex identification technique with the Oseen vortex fitting technique (Scarano et al. 1999; Carlier \& Stanislas 2005). Here, the whole flow field is first divided into vortical and non-vortical regions based on a predetermined threshold value of $\Lambda_{c i}$ (Zhou et al. 1999). Then, the measured velocity field of the vortical region is fitted to the Oseen velocity field (Scarano et al. 1999). Note that the characteristics of fitted Oseen vortex have very little dependency on the threshold value of $\Lambda_{c i}$. A fitted Oseen vortex is shown in figure 16(a) with the velocity vectors within the vortex core after the convection velocity has been subtracted from them. The relative differences in the vortex centre $\Delta c^{*}$, vortex diameter $\Delta d^{*}$ and the circulation $\Delta \Gamma^{*}$ between the Oseen vortex fitting technique and the present vortex identification technique are shown in figure $16(b)$ as a function of vorticity threshold 


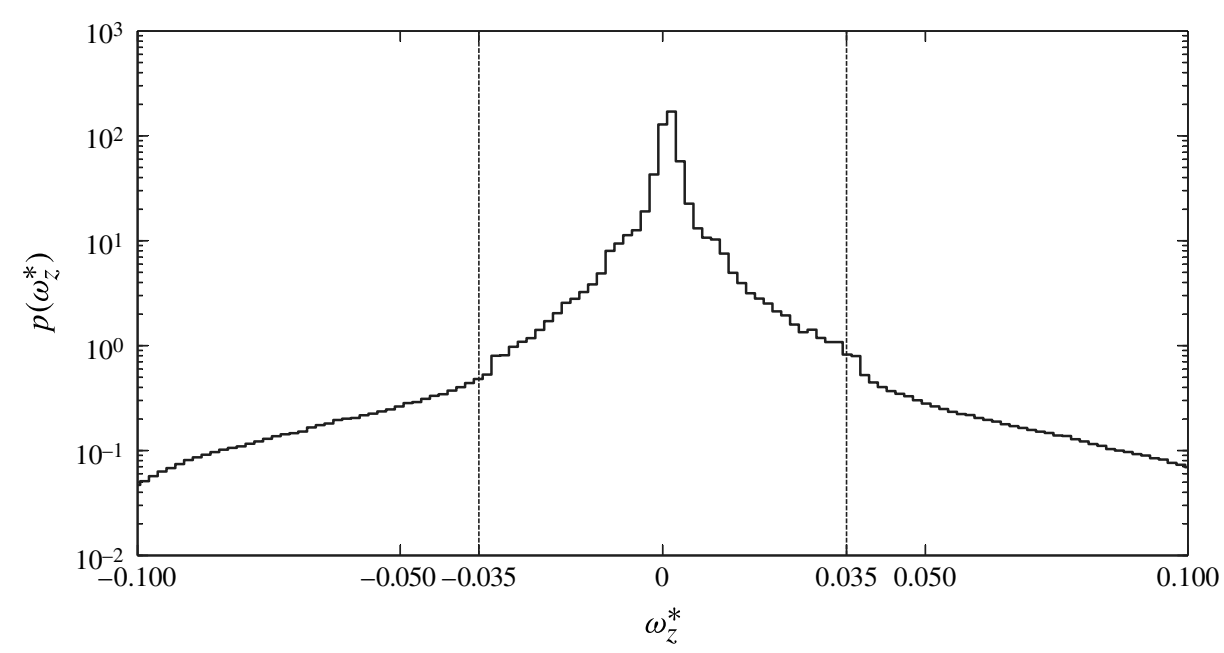

FIGURE 17. The p.d.f. of vorticity within the entire flow domain of PIV measurements.

$\omega_{z}^{*}$. Here, $\Delta \mathrm{c}^{*}, \Delta d^{*}$ and $\Delta \Gamma^{*}$ are the differences in $\mathrm{c}^{*}, d^{*}$ and $\Gamma^{*}$ between the two techniques, respectively, after they are divided by the averaged values. The threshold dependency of the present vortex identification technique can be seen in figure 16(b). This is, however, understandable since the vortex size is determined by the vorticity threshold, so is the circulation contained within the vortex. On the other hand, $\Delta \mathrm{c}^{*}$ remains nearly constant for $\omega_{z}^{*} \geqslant 0.035$, suggesting that both vortex identification techniques can give similar vortex centre locations.

The above comparison demonstrates that the present technique is as reliable as the Oseen vortex fitting technique in detecting the vortex centre accurately. However, it also indicates that the diameter and the circulation of vortex identified by the present technique are dependent on the vorticity threshold value, therefore a proper choice must be made. Clearly, the threshold value of $\omega_{z}^{*}$ should be large enough to exclude the bias from the vorticity of the fishing-line wake or any other noise in the flow. At the same time, it must be small enough not to greatly underestimate the diameter and the circulation of identified vortex. Figure 17 shows the probability density function (p.d.f.) of vorticity within the entire flow domain of PIV measurements, indicating that the shoulders of p.d.f. are located at around $\omega_{z}^{*}= \pm 0.035$. In other words, the probability of finding the vortices is very small (less than $10^{-3}$ ) when $\left|\omega_{z}^{*}\right|>0.035$. This confirms that our choice of the threshold value of $\omega_{z}^{*}=0.035$ is appropriate in detecting the vortices in the present flow domain.

The main focus of the present study is on understanding the behaviour of stagnation vortex pairs in a particular experimental set-up, which should not be influenced by the choice of vorticity threshold. This argument is supported by figure 18, where the type II vortex development with different threshold values is shown and their vortex parameters compared. The result obtained by Oseen vortex fitting technique is also included in the figure for comparison. Despite some differences in the vortex parameter values, the essential behaviour in the temporal development of both $\Gamma_{p}^{*}$ and $d^{*}$ is unchanged. The Oseen vortex fitting technique shows a similar development of $\Gamma_{p}^{*}$ to the present result, but its vortex diameter is much greater at the initial stage of vortex development $\left(t^{*}<1\right)$. This may be due to a limited number of velocity 


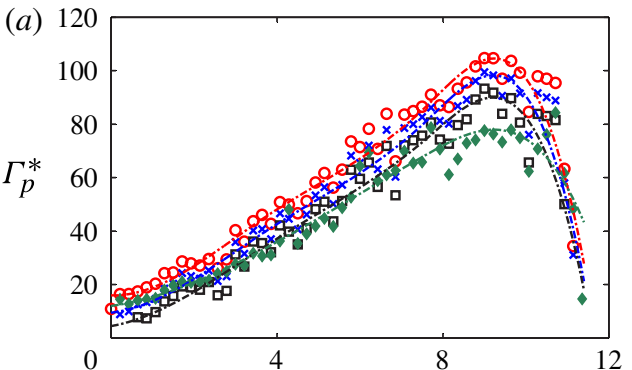

(b)
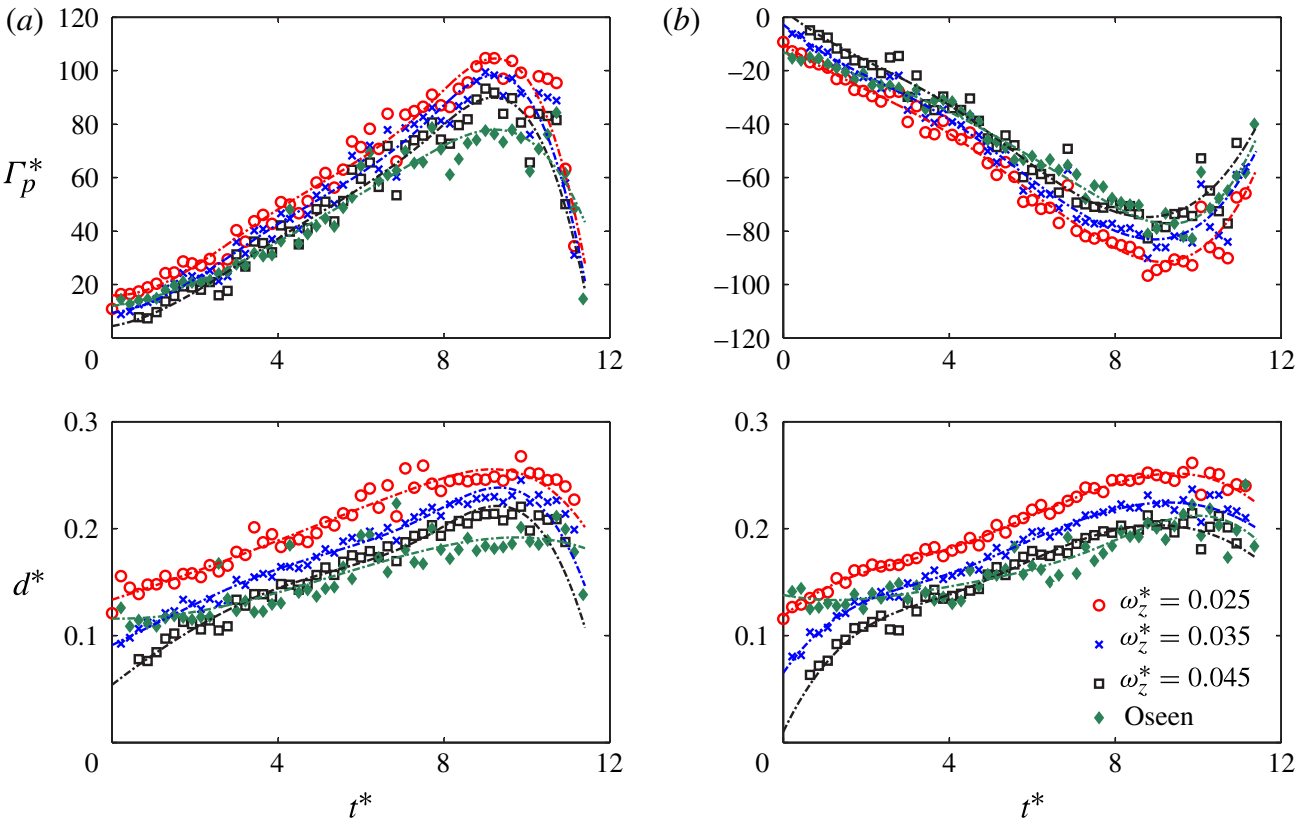

FIgURE 18. (Colour online) Temporal development of vortex parameters $\Gamma_{p}^{*}$ and $d^{*}$ with different vorticity threshold values, which is compared with the results obtained with the Oseen vortex fitting technique: $(a)$ positive vortex; $(b)$ negative vortex.

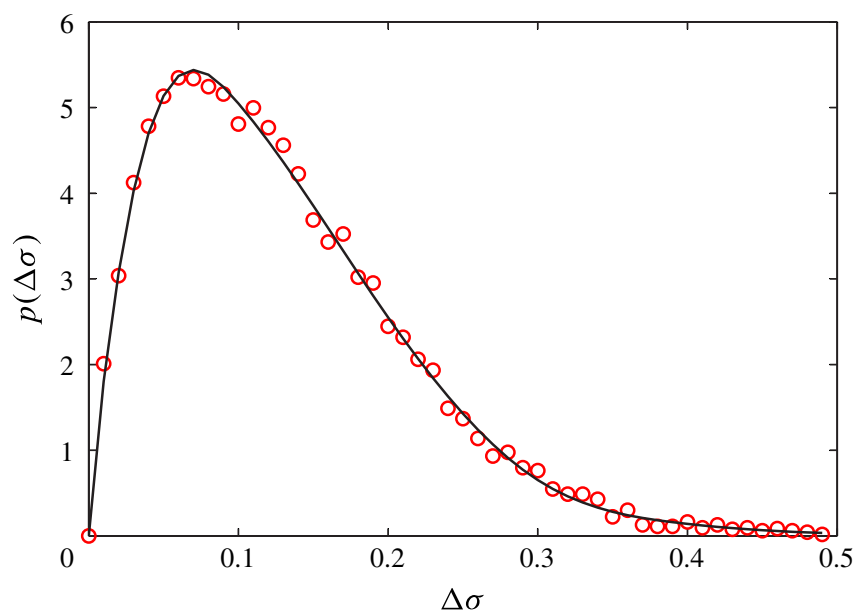

FIGURE 19. (Colour online) The p.d.f. of $\Delta \sigma$ to show the amount of error in fitting a circle to the identified vortex, where $\Delta \sigma$ is the distance between the centre of the fitted circle and the maximum vorticity location of vortex divided by their averaged distance from the wall.

vectors available for fitting a small vortex, making the Oseen vortex fitting process less reliable as compared with the present technique.

In the present study the vortex parameters, such as the size and location of the vortices, were obtained by fitting circles to the contours of vorticity concentration 


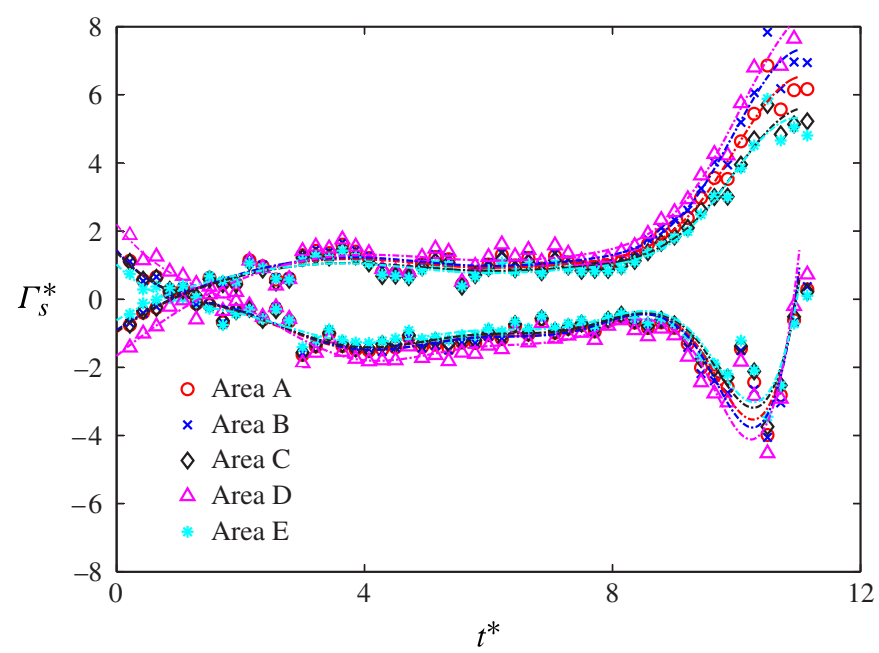

FIGURE 20. (Colour online) Temporal development of secondary circulation $\Gamma_{s}^{*}$ to show the effect of different boxes in computing vorticities.

$\begin{array}{lllll}\begin{array}{l}\text { Box } \\ \text { type }\end{array} & \begin{array}{l}\text { Height } \\ (\mathrm{mm})\end{array} & \begin{array}{l}\text { Width } \\ (\mathrm{mm})\end{array} & \begin{array}{l}\text { Area } \\ \left(\mathrm{mm}^{2}\right)\end{array} & \begin{array}{l}\text { Aspect } \\ \text { ratio }\end{array} \\ \text { A } & 10 & 2.5 & 25 & 4.0 \\ \text { B } & 12 & 2.5 & 30 & 4.8 \\ \text { C } & 8 & 2.5 & 20 & 3.2 \\ \text { D } & 10 & 3 & 30 & 3.3 \\ \text { E } & 10 & 1.5 & 20 & 6.7\end{array}$

TABLE 1. Size and shape of boxes used to compute secondary vorticities.

region using a nonlinear, least-squares fitting algorithm (Coleman \& Li 1996). The circulation of vortices was then obtained by integrating the vorticity within these circles. Figure 19 shows the p.d.f. of the relative error $\Delta \sigma$, which was obtained from an entire PIV database of the present study containing more than 12000 vortex pairs. Here, $\Delta \sigma$ is the difference between the vortex centre location of the fitted circle at the maximum vorticity location divided by their averaged distance from the wall. It can be seen in figure 19 that the p.d.f. of $\Delta \sigma$ is similar to a log-normal distribution with the peak located at around $\Delta \sigma=0.06$. The tail of the distribution is due to the non-circular vortex shape during vortical instability in the final stage of vortex development.

Secondary vorticities induced by the primary vortices over the circular plate wall were computed from the measured velocity data within a square box of $10 \mathrm{~mm}$ $(0.17 D)$ high (in the $y$ direction) and $2.5 \mathrm{~mm}(0.042 D)$ wide (in the $x$ direction), as shown by dotted lines in figures 7, 9 and 11. The present PIV measurements have a spatial resolution of $0.65 \mathrm{~mm}$ between velocity vectors, providing a $16 \times 4$ matrix of velocity data within this box. To show the effect of the box size on the computed secondary vorticities, we examine the development of secondary circulation $\Gamma_{s}^{*}$ during 
the type II development in figure 20. Here, the size and aspect ratio of five different boxes are given in table 1. Despite its small size, the present box A gives a very similar result to others $(\mathrm{B}, \mathrm{C}, \mathrm{D}$ and $\mathrm{E})$, indicating that the effect of box size used in the present study is small with a maximum error of about $20 \%$.

\section{REFERENCES}

Adrian, R. J. 2007 Hairpin vortex organization in wall turbulence. Phys. Fluids 19, 041301.

Adrian, R. J., Meinhart, C. D. \& Tomkins, C. D. 2000 Vortex organization in the outer region of the turbulent boundary layer. J. Fluid Mech. 422, 1.

Ames, F. E. \& Moffat, R. J. 1990 Heat transfer with high intensity, large-scale turbulence: the flat plate turbulent boundary layer and the cylindrical stagnation point. Technical Report HMT-43, Thermoscience Division, Mechanical Engineering Department, Stanford University, Stanford, CA.

BAE, S., LELE, S. K. \& SUNG, H. J. 2000 Influence of inflow disturbances on stagnation-region heat transfer. J. Heat Transfer 122, 258.

BAE, S., Lele, S. K. \& Sung, H. J. 2003 Direct numerical simulation of stagnation region flow and heat transfer. Phys. Fluids 15, 1462.

Barrett, M. J. \& Hollingworth, D. K. 2001 On the calculation of length scales for turbulent heat transfer correlation. J. Heat Transfer 123, 878.

Böttcher, J. \& Wedemeyer, E. 1989 The flow downstream of screens and its influence on the stagnation region of cylindrical bodies. J. Fluid Mech. 204, 501.

CARlier, J. \& Stanislas, M. 2005 Experimental study of eddy structures in a turbulent boundary layer using particle image velocimetry. J. Fluid Mech. 535, 143.

Chong, M. S., Perry, A. E. \& CAntwell, B. J. 1990 A general classification of three-dimensional flow fields. Phys. Fluids A 2, 765.

Coleman, T. F. \& LI, Y. 1996 An interior, trust region approach for nonlinear minimization subject to bounds. SIAM J. Optim. 6, 418.

Crow, S. C. 1970 Stability theory for a pair of trailing vortices. AIAA J. 8, 2172.

DANAILA, I. 2004 Vortex dipoles impinging on finite aspect ratio rectangular obstacles. Flow Turbul. Combust. 72, 391.

DhANAK, M. R. \& STUART, J. T. 1995 Distortion of the stagnation-point flow due to cross-stream vorticity in the external flow. Phil. Trans. R. Soc. Lond. A 352, 443.

Hodson, P. R. \& NAGIB, H. M. 1977 Vortices induced in a stagnation region by wakes-their incipient formation and effect on heat transfer. AIAA-Paper 77-790.

Homman, F. 1936 Der einfluss grosser zahigkeit bei der stomung um den zylinder und um die kugel. Z. Angew. Math. Mech. 16, 153.

Hunt, J. C. R., Wray, A. A. \& Moin, P. 1988 Eddies, stream and convergence zones in turbulent flows. In Proceedngs of Summer Program 1988, pp. 193-208. Center for Turbulence Research, Stanford Univesity.

Jeong, J. \& Hussain, F. 1995 On the identification of a vortex. J. Fluid Mech. 285, 69.

Kerswell, R. R. 2002 Elliptic instability. Annu. Rev. Fluid Mech. 34, 83.

Kestin, J. 1966 The effect of free stream turbulence on heat transfer rates. Adv. Heat Transfer 3, 1.

Kestin, J., Maeder, P. F. \& Sogin, H. H. 1961 The influence of turbulence on the transfer of heat to cylinders near the stagnation point. Z. Angew. Math. Phys. 12, 115.

Kestin, J. \& Wood, R. T. 1970 On the instability of two-dimensional stagnation flow. J. Fluid Mech. 44, 461.

LAPORTE, F. \& CORJON, A. 2000 Direct numerical simulations of the elliptic instability of a vortex pair. Phys. Fluids 12, 1016.

Le Dizes, S., Rossi, M. \& Moffatt, H. K. 1996 On the three-dimensional instability of elliptical vortex subjected to stretching. Phys. Fluids 8 (8), 2084.

Leweke, T. \& Williamson, C. H. K. 1998 Cooperative elliptic instability of a vortex pair. J. Fluid Mech. 360, 85. 
LIAN, Q. X. \& SU, T. C. 1994 Large vortex in front of stagnation region of a square plate induced by a fine vortex generating wire. Sci. China (Ser. A) 37, 469.

LIAN, Q. X. \& ZHOU, M. X 1989 Experimental investigation on rigid hollow hemi-spherical parachute model in accelerating and steady flows (in Chinese). Acta Aeronaut. Astronaut. Sin. 9, 84 .

Moore, D. W. \& SAFFman, P. G. 1975 The instability of a straight vortex filament in a strain field. Proc. R. Soc. Lond. Ser. A 346, 413.

Morkovin, M. V. 1979 On the question of instabilities upstream of cylindrical bodies. NASA Report 3231.

Oo, A. N. \& CHING, C. Y. 2001 Effect of turbulence with different vortical structures on stagnation region heat transfer. J. Heat Transfer 123, 665.

Oo, A. N. \& ChING, C. Y. 2002 Stagnation line heat transfer augmentation due to free stream vortical structures and vorticity. J. Heat Transfer 124, 583.

Orlandi, P. 1990 Vortex dipole rebound from a wall. Phys. Fluids A 2, 1429.

PIERREhumbert, R. T. 1986 Universal short wave instability of two-dimensional eddies in an inviscid fluid. Phys. Rev. Lett. 57, 2157.

Pirozzoli, S., Bernardini, M. \& Grasso, F. 2008 Characterization of coherent vortical structures in a supersonic turbulent boundary layer. J. Fluid Mech. 613, 205.

Robinson, S. K. 1991 Coherent motions in the turbulent boundary layer. Annu. Rev. Fluid Mech. 23, 601 .

SAdEH, W. Z. \& BRAUER, H. J. 1980 A visual investigation of turbulence in stagnation flow about a circular cylinder. J. Fluid Mech. 99, 53.

Scarano, F., Benocci, C. \& Riethmuller, M. L. 1999 Pattern recognition analysis of the turbulent flow past a backward facing step. Phys. Fluids 11 (12), 3808.

Scarano, F. \& Riethmuller, M. L. 2000 Advances in iterative multigrid PIV image processing. Exp. Fluids 29, S51-S60.

Shenoy, A. R. \& Kleinstreuer, C. 2008 Flow over a thin circular disk at low to moderate Reynolds numbers. J. Fluid Mech. 605, 253.

Stanislas, M., Perret, L. \& Foucaut, J.-M. 2008 Vortical structures in the turbulent boundary layer: a possible route to a universal representation. J. Fluid Mech. 602, 327.

SUTERA, S. P. 1965 Vorticity amplification in stagnation point flow and its effect on heat transfer. J. Fluid Mech. 21, 513.

Sutera, S. P., Maeder, P. F. \& Kestin, J. 1963 On the sensitivity of heat transfer in the stagnation-point boundary layer to free stream vorticity. J. Fluid Mech. 16, 32.

TOMKINS, C. D. \& ADRIAN, R. J. 2003 Spanwise structure and scale growth in turbulent boundary layers. J. Fluid Mech. 490, 37.

Xiong, Z. \& LELE, S. 2004 Distortion of upstream disturbances in a Hiemenz boundary layer. J. Fluid Mech. 519, 201.

Xiong, Z. \& LELE, S. 2007 Stagnation-point flow under free stream turbulence. J. Fluid Mech. 590, 1.

Zhou, J., Adrian, R. J., Balachandar, S. \& Kendall, T. M. 1999 Mechanism for generating coherent packets of hairpin vortices in channel flow. J. Fluid Mech. 387, 353. 